\title{
On Plastic Flow in Notched Hexagonal Close Packed Single Crystals
}

\author{
Balaji Selvarajou ${ }^{1}$, Babak Kondori ${ }^{2,3}$, A. Amine Benzerga ${ }^{2,3,4}$ and Shailendra P. Joshi1 ${ }^{1 *}$ \\ ${ }^{1}$ Department of Mechanical Engineering, National University of Singapore, Singapore 117576 \\ ${ }^{2}$ Department of Materials Science \& Engineering, Texas A\&M University, College Station, TX 77843 \\ ${ }^{3}$ Center for intelligent Multifunctional Materials and Structures, TEES, College Station, TX 77843 \\ ${ }^{4}$ Department of Aerospace Engineering, Texas A\&M University, College Station, TX 77843
}

April 15, 2016

\begin{abstract}
The micromechanics of anisotropic plastic flow by combined slip and twinning is investigated computationally in single crystal notched specimens. Constitutive relations for hexagonal close packed materials are used which take into account elastic anisotropy, thirty potential deformation systems, various hardening mechanisms and rate-sensitivity. The specimens are loaded perpendicular to the $c$-axis but the presence of a notch generates three-dimensional triaxial stress states. The study is motivated by recent experiments on a polycrystalline magnesium alloy. To enable comparisons with these where appropriate, three sets of activation thresholds for the various deformation systems are used. For the conditions that most closely mimic the alloy material, attention is focused on the relative roles of pyramidal $\langle c+a\rangle$ and prismatic $\langle a\rangle$ slip, as well as on the emergence of $\{10 \overline{1} 2\}[10 \overline{1} 1]$ extension twinning at sufficiently high triaxiality. In all cases, the spatial variations of stress triaxiality and plastic strain, inclusive of various system activities, are quantified along with their evolution upon straining. The implications of these findings in fundamental understanding of ductile failure of HCP alloys in general and Mg alloys in particular are discussed.
\end{abstract}

Keywords: Magnesium; Stress triaxiality; Crystal Plasticity; Material anisotropy; Failure

*Corresponding author: shailendra@nus.edu.sg 


\section{Introduction}

In materials with high crystal structure symmetry, e.g. FCC metals, plastic flow mainly occurs via dislocation mediated slip. There, the critical resolved shear stresses (CRSS) for activating different slip systems are nearly the same (Asaro and Needleman, 1985). This renders weakly anisotropic plasticity at the single crystal level. In such materials, plastic flow anisotropy may occur due to texture or grain elongation arising, for example, from severe pre-deformation during processing (Asaro and Needleman, 1985; Wenk and Van Houtte, 2004). A more fundamental origin of plastic anisotropy arises from disparate activation thresholds for various deformation mechanisms, a situation which is typical of materials with low crystal symmetry. We refer to this as inherent plastic anisotropy - the ratio of the CRSS of a slip/twin system relative to another slip/twin system. An example of this kind are materials with hexagonal close packed (HCP) crystal structure, which can deform by slip and twinning (Christian and Mahajan, 1995). In pure magnesium (Mg) the CRSS ratio of the hardest to softest slip modes is of order 100 (Kelley, 1967; Chapuis and Driver, 2011). Due to the low symmetry of the crystal structure, this type of anisotropy couples into polycrystalline texture effects.

The strong anisotropy of HCP metals such as Mg is often referred to as the origin of their damage intolerance (Bohlen et al., 2007), although it has not prevented some other HCP metal alloys, such as Zr- or Ti-based alloys, from being used as structural materials. There is a fair amount of research on characterizing plastic anisotropy in HCP metals in relation with fundamental deformation mechanisms: Ti - (Wu et al., 2008), Zr - (Lebensohn and Tomé, 1993), Mg - (Staroselsky and Anand, 2003; Agnew and Duygulu, 2005), among many others. Most studies focus, however, on uniaxial loading conditions. On the other hand, the influence of plastic anisotropy on failure under multiaxial stress states is not well understood. While this is generally relevant to textured polycrystals of high-symmetry materials, it is particularly important for low-symmetry materials. From considerations of failure micromechanics, the three-dimensional character of stress state at the current material point can be quantified by the stress triaxiality ratio, henceforth referred to simply as triaxiality, $\mathrm{T}=\sigma_{h} / \sigma_{e}$ where $\sigma_{h}$ is the hydrostatic stress and $\sigma_{e}$ is the von Mises equivalent stress. In metals, positive (tensile) triaxiality has implications on the nucleation and evolution of voids (Gurson, 1977; Benzerga and Leblond, 2010; Pineau et al., 2016). A common experimental 
approach to investigating triaxiality effects is to adopt notched specimens where various triaxiality levels can be generated by choosing appropriate notch geometries (Hancock and MacKenzie, 1976; Pineau et al., 2016). While smooth round bar specimens result in a constant $T=1 / 3$ (before necking), those with circular notches can produce $0.5 \lesssim \mathrm{T} \lesssim 1.5$ depending on the notch root radius (Needleman and Tvergaard, 1984).

An important consideration in the ductile fracture of any metallic alloy is the interplay between plastic strain and triaxiality. In particular, notched bars are ideal specimens to investigate such competition (Needleman and Tvergaard, 1984). In round notched bars, the plastic strain is usually maximum at the notch root, particularly in the early stages of straining. By way of contrast, the maximum triaxiality moves to the center of the bar after a short transient. In ductile metals a macroscopic crack typically initiates at the center of the bar, indicating a strong triaxiality effect (Hancock and MacKenzie, 1976; Needleman and Tvergaard, 1984; Hancock and Brown, 1983). Fig. 1a illustrates this situation for steel. In less ductile metals, the macroscopic crack initiates close to but away from the notch root, then eventually links to the surface in the form of a shear crack (in certain planes). This situation applies to Mg alloy AZ31, as shown in Fig. 1b. While this observation indicates that crack initiation in less ductile metals is driven by plastic strain concentrations (Alves and Jones, 1999), a mediating effect of triaxiality is not excluded. What is of particular importance, therefore, is that a fine description of plasticity in these materials is needed to develop a consistent theory of damage accumulation to fracture.

Anisotropic models coupling plasticity and damage by way of homogenization have been developed with increasing levels of sophistication (Benzerga and Leblond, 2010; Benzerga et al., 2016). In these models, anisotropic plasticity is typically represented using quadratic yield criteria (Benzerga and Besson, 2001; Keralavarma and Benzerga, 2008; Monchiet et al., 2008) or relatively simple accounts of tension-compression asymmetry (Stewart and Cazacu, 2011). Applications of such models to engineering materials remain scarce, even for high-symmetry materials, e.g., (Benzerga et al., 2004a,b; Tanguy et al., 2008). In the above applications, the net anisotropy in fracture is often traceable to void shape and spatial distribution effects. On the sole basis of experiments, it is unclear to what extent the plastic anisotropy of the matrix itself affects rates of damage accumulation. Theory, on the other hand, suggests potentially strong effects either on the basis of micromechanical finite element calculations (Benzerga and Besson, 2001; Yerra et al., 2010; Keralavarma et al., 
2011) or analytical models (Benzerga and Besson, 2001; Keralavarma and Benzerga, 2010). Despite this progress, making a definite assessment of plastic anisotropy effects in ductile damage of highsymmetry materials is subject to interpretation. On the other hand, the strong plastic anisotropy of HCP metals offers a way to decouple its effects from any morphological anisotropy effects.

Quite recently, Kondori and Benzerga (2014a) conducted notched bar experiments on hot-rolled AZ31 Mg alloy to produce multiaxial stress states. Their results revealed several characteristic features that were distinct from other anisotropic materials. First, the limit load exhibited a saturation trend upon increasing notch acuity, a behavior theretofore not reported for other materials. Second, the fracture locus (strain to failure versus triaxiality) exhibited a non-monotonic trend in contrast with other metallic alloys in the same triaxiality range. The strain to fracture was maximum at moderate values of $\mathrm{T}$ and was lowest under uniaxial loading, in strong contrast with other materials. They attributed the observed behavior to i) the change from slanted fracture in uniaxial bars to flat mode of failure in notched bars, ii) change in the damage initiation mechanism from twinninginduced microcracks under uniaxial tension to void nucleation, notably at second phase particles, followed by their growth and coalescence in notched bars; and iii) increased ease of deformation along the plate normal direction and the activation of additional deformation mechanisms, such as extension twinning, at higher triaxialities. Finally, Kondori and Benzerga (2014a) provided some details on the evolution of macroscopic plastic anisotropy in terms of the steady-state anisotropy ratio and its variation with triaxiality. Kaushik et al. (2014) conducted three-point bending experiments on notched $\mathrm{Mg}$ single crystals. Interestingly, they pointed out that profuse $\{10 \overline{1} 2\}$ extension twinning at the notch root enhances the fracture toughness.

With the above in mind, the research reported here set out to address the complex interplay of plastic anisotropy and triaxiality in HCP metals using the experiments of Kondori and Benzerga (2014a) as a primary source for modeling and comparison. To this end, the crystal plasticity simulation framework previously developed by Zhang and Joshi (2012) is employed. The framework has recently been used to model indentation in single crystal Mg (Selvarajou et al., 2014) and picked up some key aspects of the orientation dependence of pile-up and sink-in activities and their micromechanistic underpinnings, as revealed in experiments (Shin et al., 2013). Within this framework the relative contributions of slip and twinning can be explored by means of threedimensional finite element (FE) simulations of smooth and notched Mg bars. One can address 
the issue of how relative activities evolve with strain at increasing amounts of triaxiality and how such activities are spatially distributed within a notched bar. In particular, issues related to the evolution of plastic anisotropy, activation of extension twinning in notched bars loaded in-plane, as conjectured by Kondori and Benzerga (2014a), or the saturation of limit loads can be examined. Note that modeling mechanism-based failure of anisotropic materials under triaxial stress states is not attempted in this work. Coupled models of crystal plasticity and damage begin to emerge (Mbiakop et al., 2015; Han et al., 2013; Paux et al., 2015). However, available models are applicable to high-symmetry materials.

Here, attention is restricted to single crystal specimens. These act as surrogates of the real polycrystalline scenario. Indeed, rolled sheets or plates exhibit a strong basal texture; the basal planes of most grains are oriented parallel to the plate. To first order, a single crystal orientation that is appropriately aligned with the loading direction may be considered as an extreme case of such texture. In addition, the anisotropy of deformation at the polycrystalline level is directly affected by the material anisotropy at the single crystal level. Understanding the latter is therefore a necessary first step. Since the degree of inherent plastic anisotropy in $\mathrm{Mg}$ depends on the alloying composition, we consider three sets of material parameters that reflect anisotropies of pure and alloyed Mg.

\section{Formulation}

Calculations are carried out for initially round notched bars. Also, some calculations on a smooth bar are used for reference. All calculations are fully three-dimensional due to anisotropy. Three notch geometries are considered following Kondori and Benzerga (2014a) and are denoted RN $\xi$ with $\xi=10 \mathrm{R} / \phi_{0}, \mathrm{R}$ being the notch radius and $\phi_{0}$ the notch root diameter, Fig. 2. Dimensions that are kept fixed are the diameter of the cylindrical part, $\phi_{c}$, the length of the specimen, $L_{0}$, and $\phi_{0}$. Here, $\phi_{0} / \phi_{c}$ and $\phi_{c} / L_{0}$ are taken according to $3.9: 7$ and $7: 40$ ratios, respectively. Fig. 2 also shows cross-sections of the finite element meshes used. Each model is discretized into nearly 23,000 eight-node hexahedral finite elements with reduced integration.

The polycrystalline AZ31B rolled plate used by Kondori and Benzerga (2014a) had a strong basal texture. As inferred from their (0002) and (1010) pole figures, most grains have their $c$-axis 
oriented along the plate normal (or short-transverse, S) direction and their [1010] direction aligned with the transverse $(\mathrm{T})$ direction. Loading was along the rolling (or longitudinal, L) direction. To mimic the crystallographic orientation used in the experiments, the loading axis (global $z$-axis)

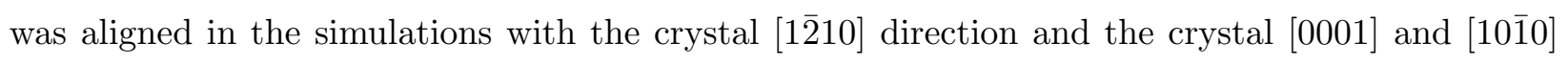
directions were aligned with the global $x$ - and $y$ - axes, respectively.

A displacement rate $\dot{U}_{y}$ was applied at the top surface of the specimen so that the nominal strain rate was $\dot{U}_{y} / L_{0}=1 \times 10^{-3} \mathrm{~s}^{-1}$. Sufficient constraints were applied on the top surface to avoid any rigid body motion. Since the applied displacement rate in all specimens is the same, the axial strain rate realized in the notch region would be specimen dependent. Yet, the very low rate sensitivity chosen below ensures that the results are independent of strain rate. To verify this, a calculation performed for the RN2 specimen at a nominal strain rate about 25 times higher revealed negligible strain rate effects.

The constitutive framework is that of rate-dependent crystal plasticity developed by Zhang and Joshi (2012) based on earlier work by Staroselsky and Anand (1998) and Kalidindi (1998). It accounts for transversely isotropic elastic response, which for $\mathrm{Mg}$ single crystals is determined by the following elastic constants (in GPa): $C_{11}=59.4, C_{12}=25.61, C_{13}=21.4, C_{33}=61.6$ and $C_{44}=$ 16.0 (Slutsky and Garland, 1957).

The velocity gradient is additively decomposed into an elastic, $\mathbf{L}^{e}$, and plastic, $\mathbf{L}^{p}$, parts:

$$
\mathbf{L}=\dot{\mathbf{F}} \mathbf{F}^{-1}=\mathbf{L}^{e}+\underbrace{\mathbf{F}^{e} \dot{\mathbf{F}}^{p} \mathbf{F}^{p-1} \mathbf{F}^{e-1}}_{\mathbf{L}^{p}}
$$

where $\mathbf{F}$ is the deformation gradient with multiplicative decomposition $\mathbf{F}^{e} \mathbf{F}^{p}$, and $\dot{\mathbf{F}}$ its material derivative. With $\mathbf{m}^{i}$ and $\mathbf{s}^{i}$ respectively denoting the current slip/twin plane normal and direction vectors for the $i^{\text {th }}$ system, the plastic velocity gradient is written as the sum of three components:

$$
\mathbf{L}^{p}=\underbrace{\left(1-\sum_{\beta=1}^{N_{t w}} f^{\beta}\right) \sum_{\alpha=1}^{N_{s}} \dot{\gamma}^{\alpha}\left(\mathbf{s}^{\alpha} \otimes \mathbf{m}^{\alpha}\right)}_{\text {slip in parent }}+\underbrace{\sum_{\beta=1}^{N_{t w}} \dot{\gamma}^{\beta}\left(\mathbf{s}^{\beta} \otimes \mathbf{m}^{\beta}\right)}_{\text {twin in parent }}+\underbrace{\left(\sum_{\beta=1}^{N_{t w}} f^{\beta}\right) \sum_{\tilde{\alpha}=1}^{N_{s}} \dot{\gamma}^{\tilde{\alpha}}\left(\mathbf{s}^{\tilde{\alpha}} \otimes \mathbf{m}^{\tilde{\alpha}}\right)}_{\text {slip in twinned region }}
$$

where $\dot{\gamma}^{i}$ denotes the shear rate on the $i^{\text {th }}$ system, $f^{\beta}$ is the twin volume fraction of the $\beta^{\text {th }}$ twin system, and $N_{s}$ and $N_{t w}$ are the total number of slip and twin systems respectively. The same 
number of slip systems is assumed in the parent and twins and the stress at a material point is taken as the weighted average of the stress in the parent and twinned regions. The Jaumann rate of Cauchy stress $\boldsymbol{\sigma}^{\nabla^{*}}$ is

$$
\boldsymbol{\sigma}^{\nabla^{*}}+\boldsymbol{\sigma}\left(\mathbf{I}: \mathbf{D}^{\mathrm{e}}\right)=\mathbf{C}: \mathbf{D}^{\mathrm{e}}
$$

where $\boldsymbol{\sigma}$ is the Cauchy stress tensor, $\mathbf{D}^{\mathrm{e}}$ the elastic rate of stretching tensor, $\mathbf{C}$ is the fourth-order elasticity tensor and $\mathbf{I}$ is the second order identity tensor. The reader is referred to Huang (1991) for detailed information on the calculation of $\boldsymbol{\sigma}^{\nabla^{*}}$ and $\mathbf{D}^{\mathrm{e}}$.

A total of thirty slip and twin systems are accounted for: 1) Three basal $\langle a\rangle$ slip systems $((0001)\langle 11 \overline{2} 0\rangle) ; 2)$ Three prismatic $\langle a\rangle$ slip systems $(\{10 \overline{1} 0\}\langle 11 \overline{2} 0\rangle) ; 3)$ Six pyramidal $\langle a\rangle$ slip systems $(\{10 \overline{1} 1\}\langle 11 \overline{2} 0\rangle)$; 4) Six pyramidal $\langle c+a\rangle$ slip systems $(\{11 \overline{2} 2\}\langle 11 \overline{2} 3\rangle)$; 5) Six extension twin systems $(\{10 \overline{1} 2\}\langle 10 \overline{1} 1\rangle)$; and 6) Six contraction twin systems $(\{10 \overline{1} 1\}\langle 10 \overline{1} \overline{2}\rangle)$. Following Zhang and Joshi (2012) constitutive relations for slip and twinning are used as follows

- Constitutive equations for slip: Each slip rate $\dot{\gamma}^{i}$ in Eq. (2) is given by a power law:

$$
\dot{\gamma}^{i}=\dot{\gamma}_{\circ}\left|\frac{\tau^{i}}{g^{i}}\right|^{m} \operatorname{sgn}\left(\tau^{i}\right)
$$

where the reference slip rate $\dot{\gamma}_{\circ}$ and rate-sensitivity exponent $m$ are material parameters, $\tau^{i}=\mathbf{m}^{i} \cdot \boldsymbol{\sigma} \cdot \mathbf{s}^{i}$ is the resolved shear stress based on the Schmid law and $g^{i}$ is the current strength of the the $i^{\text {th }}$ slip system given by

$$
g^{i}=\tau_{\circ}^{i}+\int_{t_{\circ}}^{t_{i}}\left(\dot{g}_{s l-s l}^{i}+\dot{g}_{t w-s l}^{i}\right) \mathrm{d} t
$$

Here, $\tau_{\circ}^{i}$ denotes the critical resolved shear stress (CRSS). Also, $\dot{g}_{s l-s l}^{i}$ and $\dot{g}_{t w-s l}^{i}$ are hardening terms due to slip-slip and twin-slip interactions, respectively. The former is governed by

$$
\dot{g}_{s l-s l}^{i}=\sum_{j=1}^{N_{s}} h_{i j}(\bar{\gamma}) \dot{\gamma}^{j}, \quad h_{i j}= \begin{cases}h(\bar{\gamma}) & (i=j) \\ q h(\bar{\gamma}) & (i \neq j)\end{cases}
$$


where $\bar{\gamma}$ is the cumulative slip and $1 \leq q \leq 2$. The functions $h(\bar{\gamma})$ and $q h(\bar{\gamma})$ thus represent self and latent hardening. For basal slip, a linear non-saturation type hardening law is employed while non-basal slip follows a saturation type hyperbolic hardening function. One constitutive choice is

$$
h(\bar{\gamma})= \begin{cases}h_{\circ}, & \text { (basal slip) } \\ h_{\circ}^{i} \operatorname{sech}^{2}\left|\frac{h_{\circ}^{i} \bar{\gamma}}{\tau_{s}^{\mathrm{i}}-\tau_{\circ}^{\mathrm{i}}}\right|, & \text { (non-basal slip) }\end{cases}
$$

where $h_{\circ}^{i}$ is the initial hardening modulus and $\tau_{s}^{i}$ is the saturation stress of the $i^{\text {th }}$ slip system. The twin-slip interaction hardening term $\dot{g}_{t w-s l}^{i}$ will be specified below.

- Constitutive equations for twinning: The rate of plastic shear $\dot{\gamma}^{\beta}$ on the $\beta^{\text {th }}$ twin system accrues due to a change in the twin volume fraction $f^{\beta}$ via

$$
\dot{\gamma}^{\beta}=\gamma^{t w} \dot{f}^{\beta}
$$

where $\gamma^{t w}$ is the twinning shear. For Mg the theoretical values of $\gamma^{t w}$ for extension twinning (ET) and contraction twinning (CT) are 0.129 and 0.138 , respectively. The evolution of twin volume fraction in the parent region is assumed to follow Schmid law and is described by power laws for both systems

$$
\dot{f}^{\beta}=\left\{\begin{array}{l}
\dot{f_{e t}^{\circ}}\left(\frac{\tau^{\beta}}{s_{e t}^{\beta}}\right)^{m_{t}} \\
\dot{f}_{c t}^{\circ}\left(\frac{\tau^{\beta}}{s_{c t}^{\beta}}\right)^{m_{t}}
\end{array}\right.
$$

where $\dot{f}_{\text {et }}^{\circ}$ and $\dot{f}_{c t}^{\circ}$ are average reference values, $m_{t}$ is the rate-sensitivity exponent for twinning, and $s^{\beta}$ is the current strength of the $\beta^{\text {th }}$ twin system given by

$$
s^{\beta}=\tau_{\circ}^{\beta}+\int_{t_{\circ}}^{t_{i}}\left(\dot{s}_{t w-t w}^{\beta}+\dot{s}_{s l-t w}^{\beta}\right) \mathrm{d} t
$$

where $\tau_{\circ}^{\beta}$ is the CRSS for twin system $\beta, \dot{s}_{t w-t w}^{\beta}$ is the hardening due to twin-twin interactions and $\dot{s}_{s l-t w}^{\beta}$ is the hardening due to slip-twin interactions. Hardening due to twin-twin 
interaction follows a hyperbolic function

$$
\dot{s}_{t w-t w}^{\beta}=h_{\mathrm{et}}^{\beta} \operatorname{sech}^{2}\left|\frac{h_{\mathrm{et}}^{\beta} \bar{\gamma}_{e t}}{\tau_{s_{-} e t}^{\beta}-\tau_{\mathrm{o}_{-} e t}^{\beta}}\right| \dot{\gamma}^{\beta}
$$

for extension twinning and a non-saturating function

$$
\dot{s}_{t w-t w}^{\beta}=H_{c t}\left(\sum_{\kappa=1}^{N_{c t}} f^{\kappa}\right)^{b} \dot{\gamma}^{\beta}
$$

for contraction twinning. In Eq. (11) $\bar{\gamma}_{e t}$ denotes the total shear strain on all ET systems. Also, the initial hardening modulus $h_{e t}^{\beta}$ and the saturation stress $\tau_{s_{-} e t}^{\beta}$ are material parameters. Likewise, in Eq. (12) $H_{c t}$ and $b$ are hardening parameters, $N_{c t}$ being the total number of CT systems. Following Zhang and Joshi (2012) hardening of the twin systems is taken to be independent of slip so that $\dot{s}_{s l-t w}^{i}=0$ in Eq. (10). On the other hand, twinning is assumed to affect the slip hardening, and the term $\dot{g}_{t w-s l}^{i}$ in Eq. (5) is given by

$$
\dot{g}_{t w-s l}^{\beta}=\left\{\begin{array}{l}
h_{e t_{s} s l}^{\beta} \operatorname{sech}^{2}\left|\frac{h_{e t_{-} s l}^{\beta} \bar{\gamma}_{e t}}{\tau_{s_{-} e t}^{\beta}-\tau_{o_{-} e t}^{\beta}}\right| \dot{\gamma}^{\beta} \\
0.5 H_{c t_{-} s l}\left(\bar{\gamma}_{c t}\right)^{-0.5} \dot{\gamma}_{c t},
\end{array}\right.
$$

with parameters defined analogous to the above. Although not directly evident in Eq. (2), double twinning is accommodated in the formulation as follows. For a particular twin mode (extension twinning versus contraction twinning), when the total twin volume fraction reaches a critical value $f_{c}$ (set equal to 0.9 ) the lattice is rotated to the twinned orientation instantaneously. Specifically, the new orientation is that of the twin system with the largest $f^{\beta}$ value (Tomé et al., 1991). After reorientation, deformation systems are reset accordingly and resolved stresses are calculated using their new orientation. Any twinning that would occur in the reoriented region would de facto correspond to double twinning.

The above constitutive model has been implemented as a User MATerial (UMAT) subroutine in ABAQUS/STANDARD ${ }^{\circledR}$ (Systèmes, 2012). For algorithmic and other details the reader is referred to (Zhang and Joshi, 2012). 


\section{Results and Discussion}

The material parameters entering Eqs. (4) through (13) are provided in Table 1 and are representative of pure Mg. Also, $\dot{\gamma}_{\circ}=0.001 \mathrm{~s}^{-1}, m=50$ and $q=1$ for all slip systems; and $\dot{f}_{\text {et }}^{\circ}=0.001 \mathrm{~s}^{-1}$, $\dot{f}_{c t}^{\circ}=0.0001 s^{-1}$ and $m_{t}=50$. Two other sets of parameters are used which are representative of alloys. Table 2 gathers actual and normalized CRSS values used alongside their counterparts for pure Mg from Table 1. Of these, alloy-2 closely represents CRSS ratios of AZ31B (Lou et al., 2007; Hong et al., 2010). Note that the CRSS ratio of the softer basal slip system is increased while the CRSS ratio of the harder pyramidal $\langle c+a\rangle$ slip system is reduced in both alloys. For simplicity, the same hardening rates of pure $\mathrm{Mg}$ are retained in the alloys.

The overall mechanical responses are given in terms of the normalized load $\left(s=F / A_{0}\right)$ versus normalized diametric reduction $\left(\delta_{\alpha}=\Delta \phi_{\alpha} / \phi_{0}\right)$ where $F$ is the total force along the loading direction, $A_{0}=\pi / 4 \phi_{0}^{2}$ is the initial cross-sectional area at the notch root, and $\Delta \phi_{\alpha}$ is the reduction in diameter along direction $\alpha$ (either $\mathrm{T}$ or $\mathrm{S}$ ). Also, the net total strain is given by

$$
\varepsilon_{\mathrm{A}}=\varepsilon_{[0001]}+\varepsilon_{[10 \overline{10}]}
$$

where $\varepsilon_{\alpha}=\ln \left(\phi_{\alpha} / \phi_{0}\right)$ is the net strain in lateral direction $\alpha$. Following Kondori and Benzerga (2014a), the strain anisotropy ratio is defined as:

$$
\mathrm{R}_{\mathrm{L}}=\varepsilon_{[10 \overline{1} 0]} / \varepsilon_{[0001]}
$$

The calculations were terminated at a displacement $U_{y} / L_{0}=0.20$ in the smooth bar and $U_{y} / L_{0}=$ 0.02 in the notched bars. These values correspond to $\varepsilon_{\mathrm{A}}=0.2$ in the smooth bar and $\varepsilon_{\mathrm{A}}=0.3$ in the notched bars. Values of $\varepsilon_{\mathrm{A}}$ reported after necking are taken at the neck.

\subsection{Deformation of pure $\mathrm{Mg}$}

\subsubsection{Overall Mechanical Responses}

Fig. 3 shows the load versus diameter reduction responses of the notched and smooth bars. The limit load (maximum load attained) increases with increasing notch acuity, as expected based on increasing the triaxiality. Moreover, it is immediately clear that the smooth and RN10 bars have a 
greater propensity to deform along $[10 \overline{1} 0]=\mathrm{T}$ (Fig. 3b) compared with $[0001]=\mathrm{S}$ (Fig. 3a) while the RN4 and RN2 bars exhibit more ease to deform along [0001]. The deformation anisotropy is intense in the smooth specimen: the load drops precipitously with very small diametric reduction along the [0001] direction, while the same load drop extends over a wider range of diametric reduction in the $[10 \overline{10}]$ direction. In the smooth specimen, strain localization occurs and a neck develops as a result of modeling the loading ends (see the mesh of Fig. 2).

The deformation anisotropy may be characterized by $\mathrm{R}_{\mathrm{L}}$ in Eq. (15). Fig. 4 shows the evolution of $R_{L}$ with the net strain measure $\varepsilon_{A}$ in Eq. (14). Since definition (15) includes contributions from elastic strains the transient response is affected by the elastic-plastic transition, which eventually reflects in a non-monotonic evolution of $R_{L}$ (the initial value of $R_{L}$ is 1.54 due to elastic anisotropy). After a transient, the values of $\mathrm{R}_{\mathrm{L}}$ are significantly higher in the smooth specimen compared to the notched specimens and those of the RN4 and RN2 bars are closer together. Fig. 5 shows the computed $R_{\mathrm{L}}$ values and sketched cross-sections of the original and deformed specimens at $\varepsilon_{\mathrm{A}}=0.1$. In all cases $R_{L}>1$, which means that initially circular cross-sections have become oval with the major and minor axes along [0001] and [1010], respectively.

\subsubsection{Triaxiality Distribution in the Notch}

Figure 6 shows contour plots of triaxiality $\mathrm{T}$ in the $\mathrm{RN} 10$ and $\mathrm{RN} 2$ specimens at $\varepsilon_{\mathrm{A}}=0.1$. As expected based on anisotropy, the distribution of $T$ varies from one longitudinal section to the other. In views that contain the $[0001]=\mathrm{S}$ direction, the triaxiality is maximum at the center, as expected for plastically isotropic (Alves and Jones, 1999) or even moderately anisotropic materials Benzerga et al. (1999). This is better quantified in Fig. 6c (left plots) at various overall strain levels. On the other hand, in views that contain the $[10 \overline{1} 0]=\mathrm{T}$ direction, the maximum triaxiality is attained away from the center, at $\eta_{[10 \overline{1} 0]} \sim \pm 0.25-0.35$ (depending on strain level) where $\eta_{\alpha}=r_{\alpha} / \phi_{\alpha}$ refers to the radial position normalized by the current notch root diameter along $\alpha$. This trend is stronger in the RN2 specimen, as quantified in Fig. 6c (right plots). Other features are not so typical of isotropic materials. Pockets of relatively high triaxiality are for instance noted in the L-S view of the RN10 specimen away from the equator (Fig. 6a). In fact, Kondori and Benzerga (2014a) reported surface cracks at such locations. Furthermore, the location of the absolute maximum triaxiality in the L-S view of the RN2 specimen is not in the equator, as probed quantitatively in Fig. 6c, but above and 
below it (Fig. 6b).

In some respects, therefore, the triaxiality distributions are quite distinct from commonly expected trends. They are induced by the strong plastic anisotropy of the pure $\mathrm{Mg}$ single crystals.

\subsubsection{Deformation Micromechanics}

In a smooth single crystal specimen subjected to tensile loading along [12 10$]$ (fig. 2a), the mechanisms available to accommodate plastic deformation are: (i) pyramidal $\langle c+a\rangle$ slip and $\{10 \overline{1} 1\}\langle 10 \overline{1} \overline{2}\rangle$ (contraction) twinning for contraction along [0001], (ii) prismatic $\langle a\rangle$, pyramidal $\langle a\rangle$, pyramidal $\langle c+a\rangle$ slip and $\{10 \overline{1} 1\}\langle 10 \overline{1} \overline{2}\rangle$ (contraction) twinning for contraction along [10ㅍ], and (iii) prismatic $\langle a\rangle$, pyramidal $\langle a\rangle$, pyramidal $\langle c+a\rangle$ slip and $\{10 \overline{1} 1\}\langle 10 \overline{1} \overline{2}\rangle$ (contraction) twinning for extension in the loading direction [1 $\overline{2} 10]$. In comparison, highly non-homogeneous stress states in notched specimens are expected to complicate the plastic flow process. The micromechanics that governs this process can be analyzed by examining the volume averaged relative activity, $\bar{\zeta}^{i}$, of each slip or twin system

$$
\bar{\zeta}^{i}=\frac{\int_{V} \dot{\gamma}^{i} d V}{\int_{V} \dot{\Gamma} d V}, \quad \dot{\Gamma}=\sum_{\alpha=1}^{N} \dot{\gamma}^{\alpha}
$$

where $\dot{\Gamma}$ represents the total shear rate due to all deformation systems. Averaging is carried over the whole gauge section in smooth bars and the notched region only in notched ones.

Fig. 7 shows the evolution of $\bar{\zeta}^{\alpha}$ for the slip and twin systems for the smooth, RN10 and RN2 specimens. The relative activities for the RN4 specimens show consistent trend and are omitted here for brevity. In the smooth specimen (Fig. 7a) prismatic $\langle a\rangle$ slip dominates, as expected. No other deformation mechanism is readily available to accommodate contraction along the $c$-axis. Thus, in the absence of elasticity, one would expect an infinite value of $R_{L}$, which is the trend seen at large strains in Fig. 4. The drop in $\mathrm{R}_{\mathrm{L}}$ at $\varepsilon_{\mathrm{A}} \sim 0.02$ is due to the brief activity of contraction twinning to accommodate $\varepsilon_{[0001]}$. However, due to its non-saturation type hardening and high hardening rate (Eq. 12), subsequent evolution of contraction twinning becomes progressively difficult. Though pyramidal $\langle c+a\rangle$ slip systems possess higher Schmid factor and lower CRSS compared to contraction twinning systems, its activation is precluded due to latent hardening from the strain accrued on 
the prismatic $\langle a\rangle$ system. On the other hand, the model does not consider prior latent hardening of contraction twin systems (a physical basis of this assumption is discussed in Zhang and Joshi (2012)) and therefore, it is preferentially activated, albeit only over a short range of strain. This effect implies that the deformation anisotropy depends not only on the self-hardening characteristics of relevant slip/twin systems, but also on the latent hardening between various slip and twin systems.

By way of comparison, the relative activity evolution in notched specimens is more complex (Fig. 7b-7c). While prismatic $\langle a\rangle$ slip continues to be an important deformation mode for accommodating plastic deformation along [10̄0], both RN10 (Fig. 7b) and RN2 (Fig. 7c) specimens exhibit increasing influence of pyramidal $\langle c+a\rangle$ slip that accommodates plastic deformation along [0001]. Note that despite the presence of latent hardening, pyramidal $\langle c+a\rangle$ slip does activate in notched specimens. Two factors lead to an increase in the resolved shear stress (RSS) for pyramidal $\langle c+a\rangle$ slip. First, the overall normalized load carried by the notched specimens is higher than the load in smooth specimen (Fig. 3). Second, in the notch specimens, lateral stresses arise along [0001] $\left(\sigma_{[0001]}\right)$ and $[10 \overline{1} 0]\left(\sigma_{[10 \overline{1} 0]}\right)$. Fig. 8a-8b and Fig. 8c- 8d show the contours of the lateral normal stresses in the RN10 and RN2 specimens respectively at $\varepsilon_{\mathrm{A}}=0.1$. In the smooth specimen, tensile stress along [1210] contributes to positive RSS on the pyramidal $\langle c+a\rangle$ slip planes. In the notch specimens, in addition to the tensile stress along [12 10$]$, compressive stress along the $c$-axis (negative $\sigma_{[0001]}$ restricted to a small region near the notch root) and tensile stress along [10 $\left.\overline{1} 0\right]$ (positive $\left.\sigma_{[10 \overline{1} 0]}\right)$ enhance the RSS on the pyramidal $\langle c+a\rangle$ slip planes in the notch regions. These factors lead to the activity of pyramidal $\langle c+a\rangle$ slip in the notched specimens though latent hardening prevents its activity in the smooth specimen. Owing to the triaxial stress state, basal slip, pyramidal $\langle a\rangle$ slip, contraction twinning and extension twinning modes are also activated at various stages of deformation. Moreover, the influence of these secondary deformation modes is enhanced with increasing triaxiality (Fig. 7c). This dependence of relative slip and twin system activity on the triaxiality has a remarkable effect on the evolution of the deformation anisotropy, as seen in Fig. 5. The overall lowering of $R_{L}$ with increasing stress triaxiality results from the activation of pyramidal $\langle c+a\rangle$ slip along [0001] relative to the prismatic $\langle a\rangle$ slip along [10̄10].

The preceding results provide insight into the average mechanical response. In particular, the relative activities of the underlying slip and twinning systems are averaged over the specimens. It 
is also useful to examine how the presence of a notch influences the spatial distribution of these deformation modes. This information is important because notch induced triaxiality activates different deformation systems that otherwise may not co-exist under uniaxial stress conditions. For instance, pyramidal $\langle c+a\rangle$ slip and extension twinning do not co-exist under uniaxial loading of single crystals. The ensuing slip-twin and twin-twin interactions may have implications on the post-yield behavior, including softening and fracture (Yoo, 1981). In the following, we discuss the micromechanics of slip and twinning modes. For brevity, all the results are presented at a fixed areal strain of $\varepsilon_{\mathrm{A}}=0.1$.

i. Total plastic strain: Fig. 9 shows the total accumulated plastic strain $\left(\Gamma=\int_{0}^{t} \sum_{\alpha=1}^{N} \gamma^{\alpha}\left(t^{\prime}\right) \mathrm{d} t^{\prime}\right.$ where $t$ is time-like parameter) in the smooth, RN10 and RN2 specimens viewed along the [0001] and $[10 \overline{1} 0]$ directions. In the smooth specimen, the presence of the specimen grips and the waist region leads to an inhomogeneous stress state akin to the stress state in the experiments. At fixed $\varepsilon_{\mathrm{A}}$, the gradient of plastic strain is steeper in the RN2 specimen. Also, the plastic zone is restricted to the notch region in the RN10 specimen whereas it extends well beyond the notch in the RN2 specimen. Further, for a given notched specimen, $\Gamma$ values are larger along [0001] than along $[10 \overline{1} 0]$.

The complex distribution of triaxiality discussed in section 3.1.2 motivates an inspection of the notch effects on the distribution of the equivalent plastic strain. This is important because, evolution of damage (e.g. voids) is usually described in terms of the equivalent plastic strain $\left(\varepsilon_{\mathrm{eq}}^{p}\right)$ along with triaxiality (Rice and Tracey, 1969). To this end, Fig. 10 shows the variation of $\varepsilon_{\mathrm{eq}}^{p}$ along [0001] and [10̄0] in the minimum section of the notch specimens where $\left(\varepsilon_{\mathrm{eq}}^{p}=\sqrt{\frac{2}{3} \varepsilon_{i j}^{p} \varepsilon_{i j}^{p}}\right)$. Here, $\varepsilon_{i j}^{p}$ are the components of the plastic strain tensor, $\varepsilon^{p}=\int_{0}^{t} \mathbf{D}^{p} \mathrm{~d} t^{\prime}$, with $\mathbf{D}^{p}$ being the symmetric part of $\mathbf{L}^{p}$. In RN10 specimen, the difference between the maximum (occurs at the notch root) and the minimum (occurs at the notch center) equivalent plastic strain along [0001] is small resulting in a nearly uniform distribution. Further, it remains so with increasing $\varepsilon_{\mathrm{A}}$. Likewise, the variation along [1010] is also small, although the peak occurs at a distance $\sim 0.2 \phi_{[10 \overline{1} 0]}$ while the minimum is still at the notch center. In RN2 specimen, the $\varepsilon_{\mathrm{eq}}^{p}$ distribution trends are similar along [0001] and [1010] with the maximum at notch root and minimum at the notch center. Note that the plastic strain gradient is larger in 
RN2 compared to RN10 indicating larger strain concentration at the notch root.

ii. Prismatic $\langle a\rangle$ slip: Fig. 11 shows that prismatic $\langle a\rangle$ slip is active in both smooth and notched specimens. Only two of the three variants accrue slip as the variant with the slip plane parallel to the loading direction cannot be active. In notched specimens, the peak plastic strain on these slip systems is preferentially located close to the notch root along [10ㅅ] and its magnitude is larger in the RN2 specimen. Interestingly, it was noted earlier that the relative activity of prismatic $\langle a\rangle$ slip decreases with increasing triaxiality (cf. Fig. 7). As the relative activity depends on the product of material volume of interest and the amount of slip in that volume, increasing triaxiality implies two things: 1) The volume over which prismatic $\langle a\rangle$ slip occurs decreases, and 2) other slip/twin modes become increasingly important, both in terms of magnitude and their spatial distribution.

iii. Basal slip: In the smooth specimen, the Schmid factors of all three basal systems are identically zero. On the other hand, in notched specimens the stress concentration at the intersection of the notched region with the rest of the uniform region appears to introduce sufficiently high local RSS on the basal systems causing them to be active (Fig. 12). The basal slip system shown here is one with the Burger's vector parallel to the loading direction. The slip on this slip system is an order of magnitude higher than the other two basal slip systems (not shown for brevity).

iv. Pyramidal $\langle c+a\rangle$ slip: For reasons discussed earlier in the section, pyramidal $\langle c+a\rangle$ slip is inactive in the smooth specimen despite of having the highest Schmid factor. Fig. 13 shows the pyramidal $\langle c+a\rangle$ slip in the RN10 and RN2 specimens. In the notched specimens, strain is accrued on two of the six pyramidal $\langle c+a\rangle$ slip variants where the Burger's vector, the slip plane normal and the loading direction are coplanar.

v. Twinning: Contraction twinning activity is evident in both smooth and notch specimens (Fig. 7). On the other hand, extension twinning is inactive in the smooth specimen, because the $c$-axis is under contraction. In the notched specimens however, the lateral stresses along [0001] and [1010] vary from compression to tension within the notch region (Fig. 8); as a result, the stress state in this region favors extension twinning at some locations and contraction 
twinning at other locations. Although both, extension and contraction twinning are active in the notched specimens, the accrued volume fraction (v.f.) is below the critical twin v.f. $\left(f_{c}\right)$ set as the criterion for twin induced lattice reorientation in this work, at least up to $\varepsilon_{\mathrm{A}}=0.1$. In fact, for the material parameters considered in this set of simulations the twinning reorientation criterion is not satisfied up to $\varepsilon_{\mathrm{A}} \sim 0.4$. The underpinnings for low twin v.f.'s are different in the case of extension and contraction twinning. Given the polar nature of twinning, twin v.f. evolves as a function of the effective RSS on twin systems, which depends delicately on the intensity of triaxial stress state within the notch. It turns out that this stress state results in a positive RSS along $\{10 \overline{1} 2\}\langle 10 \overline{1} 1\rangle$, but is small and evolves slowly so that the total extension twin v.f. remains below the critical value over a large range of applied strain. On the other hand, the RSS on $\{10 \overline{1} 1\}\langle 10 \overline{1} 2\rangle$ is sufficiently high for contraction twinning, but the nonsaturation type hardening of these twin systems results in low twin v.f. The role of (extension) twinning will be discussed further below.

\subsection{Effect of Inherent Plastic Anisotropy: Mg Alloys}

The pure $\mathrm{Mg}$ analyses presented in the previous section have provided some qualitative trends that already corroborate some experimental findings. However, pure Mg single crystals possess high plastic anisotropy (Zhang and Joshi, 2012; Raeisinia et al., 2011) compared with their alloyed counterparts even at dilute concentrations (Akhtar and Teghtsoonian, 1969a,b).

This raises the issue of the influence of plastic anisotropy on the micromechanics of deformation as well as other macroscopic indicators such as limit loads and anisotropy ratios. Agnew and Duygulu (2005) noted that the ratio of the CRSS of pyramidal $\langle c+a\rangle$ to prismatic $\langle a\rangle$ slip influences the deformation anisotropy in AZ31 when loaded along both the transverse and rolling directions. Following their observations, the pyramidal $\langle c+a\rangle$ CRSS in the two alloy cases were modified while keeping the remaining CRSS values the same, see Table 2. First, we compare the diametric reduction $\left(\delta_{[0001]}\right)$ as a function of overall stress triaxiality in the pure $\mathrm{Mg}$, alloy-1 and alloy-2 specimens together with the experimental results of Kondori and Benzerga (2014b) (Fig. 14). The experimental result is known at areal strain corresponding to fracture initiation $\left(\varepsilon_{\mathbf{A}}^{\mathrm{f}}\right)$, which is indicated in the figure and the simulation results are presented at the same strain, although our model does not model fracture. As pointed out by Kondori and Benzerga (2014a), the experimental 
trend is non-monotonic with the highest fracture initiation strain for RN10 specimen. The alloy 2 simulations capture this trend while the pure $\mathrm{Mg}$ and alloy-1 simulations fail to do so. Note that in terms of the CRSS ratios the plastic anisotropy of alloy-1 is not significantly different from that of alloy-2. The remarkable difference between the two alloy cases is particularly interesting as it highlights the marked influence of plastic anisotropy.

Fig. 15 compares the limit load measured from the experiments and the load values obtained in the simulations at strain corresponding to the experimental limit load. The experiments show an increase in limit load with increasing triaxiality; the increase in limit load between RN10 and RN4 is smaller than the increase observed between the smooth and RN10 specimens. This weaker increase in the limit load with increasing triaxiality is captured in the alloy-2 specimens. The limit load depends on the plastic flow anisotropy, self-hardening of slip/twin systems, latent-hardening between various slip/twin systems and the stress state triaxiality. The simulations capture the qualitative trend of weaker dependence of load on triaxiality at higher triaxiality; quantitative match with experiments is possible only if the hardening rates of the slip/twin systems are modified to represent AZ31B (as noted earlier in this section, the hardening rates of pure $\mathrm{Mg}$ are retained for the alloys).

Fig. 16 shows the trends of $\mathrm{R}_{\mathrm{L}}$ with $\varepsilon_{\mathrm{A}}$ for the two alloy cases (Fig. 16a) and as a function of stress triaxiality at experimentally reported fracture initiation strain $\left(\varepsilon_{\mathrm{A}}^{\mathrm{f}}\right)$, see Fig. 16b. Comparing Fig. 16a with its pure Mg counterpart (Fig. 4) reveals several insights. First, for a given triaxiality a reduced CRSS anisotropy leads to a monotonic reduction in the deformation anisotropy. Second, there is a qualitative change in the manner in which $R_{L}$ evolves in smooth specimens for the two alloy cases. While the alloy-1 exhibits a tempered but similar trend as in pure $\mathrm{Mg}$, in alloy-2, $\mathrm{R}_{\mathrm{L}}$ continues to decrease beyond the initial peak. The latter implies that the deformation along the [0001] evolves more rapidly compared to the deformation along [1010]. Third, the simulations of alloy cases predict a transition of $R_{L}>1$ to $R_{L}<1$ with increasing triaxiality, which is consistent with the experiments of Kondori and Benzerga (2014a), as seen in Fig. 16b. Moreover, the $\mathbf{R}_{\mathbf{L}}$ values for alloy-2 compare well with the experimentally reported values over the range of stress triaxiality. 


\subsubsection{Distribution of Triaxiality in Notch Regions}

Fig. 17 shows the variation of triaxiality along [0001] and [10피 in the minimum cross-section at different $\varepsilon_{\mathrm{A}}$ in alloy-2 single crystals. When compared with pure Mg (Fig. 6c), strong qualitative similarities are noted: in the RN10 specimen, along both [0001] and [1010], $\mathrm{T}_{\min }$ and $\mathrm{T}_{\max }$ occur at the notch root and at the center of the notch, respectively. The non-monotonic distribution in the RN2 specimen is also similar to the pure Mg case. This non-monotonic behavior is observed along both [0001] and [1010] though the effect is much more pronounced along [1010]. As in the pure $\mathrm{Mg}$ case, $\mathrm{T}_{\max }$ is located at $\sim 0.25 \phi_{[10 \overline{10}]}$ from the notch root. Although not shown here, the non-monotonic behavior also persists in RN2 specimens of alloy-1 single crystal.

\subsubsection{Deformation Micromechanics of $\mathrm{Mg}$ Alloys}

Fig. 18 compares the relative activity evolution of all slip and twin systems in alloy-2 for the smooth, RN10 and RN2 specimens. The results for alloy-1 case follow the same trend and are omitted here for brevity. In smooth specimen, in addition to the prismatic $\langle a\rangle$ slip activity, pyramidal $\langle c+a\rangle$ slip contributes to the overall plasticity. This is distinct from the pure $\mathrm{Mg}$ smooth specimen where pyramidal $\langle c+a\rangle$ is absent altogether (cf. Fig. 7a). Although the pyramidal $\langle c+a\rangle$ slip activity is significant, it is still the second most active deformation system despite having the highest Schmid factor; the CRSS of prismatic $\langle a\rangle$ slip is lower compared to pyramidal $\langle c+a\rangle$ slip. The maximum strength (the saturation stress) that the prismatic $\langle a\rangle$ slip systems attain before their hardening saturates is also lower than the maximum strength that the pyramidal $\langle c+a\rangle$ slip systems attain (Table 2). The higher relative activity of prismatic $\langle a\rangle$ slip is attributed to the lower CRSS and saturation stress of prismatic $\langle a\rangle$ slip systems. For $0.04 \leq \varepsilon_{\mathrm{A}} \leq 0.12$, the $\mathrm{R}_{\mathrm{L}}$ remains nearly constant as the prismatic $\langle a\rangle$ slip and pyramidal $\langle c+a\rangle$ slip activities are also constant.

In notched specimens, pyramidal $\langle c+a\rangle$ slip is as important as the prismatic $\langle a\rangle$ slip. Its contribution increases with increasing triaxiality; in fact, in the RN2 case, it contributes more to the total plasticity than any other deformation mechanism, which explains the drop in $\mathrm{R}_{\mathrm{L}}$ below 1 . The basal and pyramidal $\langle a\rangle$ slip modes as well as contraction twinning also make non-negligible contributions to the overall plasticity in the presence of notch and their activities also become more conspicuous with increasing triaxiality. Although extension twinning activity appears to be weak, 
lattice reorientation occurs at large strains in regions of high stress intensity.

As in the pure $\mathrm{Mg}$ case, we now discuss the spatial distribution of different slip and twinning plastic strain at a fixed strain $\varepsilon_{\mathrm{A}}=0.1$ for the alloy- 2 specimens.

1. Slip: For a given applied macroscopic strain, the plastic strain is expected to be the same irrespective of the plastic anisotropy of the material. The magnitude and distribution of the total plastic slip in the smooth and notched specimens of alloy-2 single crystal is nearly identical to the pure $\mathrm{Mg}$ specimens and therefore not repeated here. The magnitudes of prismatic $\langle a\rangle$ slip and pyramidal $\langle c+a\rangle$ slip are lower and higher respectively, compared to the pure $\mathrm{Mg}$ specimens for the entire range of triaxiality considered, but their distributions are essentially the same as in Fig. 11 and Fig. 13 respectively. Likewise, the distributions of $\varepsilon_{\text {eq }}^{p}$ along [0001] and [10̄10] are qualitatively similar to their pure Mg counterparts in Fig. 10. A key difference between the micromechanics of pure $\mathrm{Mg}$ and alloy-2 specimens is in the occurrence of twinning in the latter.

2. Extension Twinning: Uniaxial tensile stress along [1210] in the smooth specimen does not favor extension twinning. In notched specimens however, lateral stresses $\left(\sigma_{[0001]}, \sigma_{[10 \overline{1} 0]}\right)$ exist in the notch region, which can cause extension twinning (Fig. 19a). Fig. 19b-19c and Fig. 19d19e show the contours of the lateral stresses in the RN10 and RN2 specimens respectively at $\varepsilon_{\mathrm{A}}=0.1$. A noteworthy feature is that the lateral stresses range from being compressive in some regions of the notch to tensile in other regions of the notch. For extension twinning to occur, the net stress should be such that the RSS satisfies the polarity of twinning. For the present case, a tensile (positive) $\sigma_{[0001]}$ and a compressive (negative) $\sigma_{[10 \overline{1} 0]}$ will result in meeting this requirement, as indicated in Fig. 19a. Note that, although the shear stress $\sigma_{[1 \overline{2} 10]-[10 \overline{1} 0]}$ in the notch region also contributes to the RSS on the extension twin systems its magnitude is two orders of magnitude lower than $\sigma_{[0001]}$ and $\sigma_{[10 \overline{10}]}$. As such, its contribution to extension twinning is negligible.

For a given notch acuity the lateral stresses at a fixed $\varepsilon_{\mathrm{A}}$ are higher in alloy- 2 specimen compared to the pure Mg specimen, because of the higher CRSS values for the alloy. As a 
result, the alloy-2 specimen has higher propensity to twinning than its pure Mg counterpart. Moreover, for the alloy-2 case the lateral stresses are higher for RN2 (at fixed $\varepsilon_{\mathrm{A}}$ ) than RN10 and correspondingly its twinned volume is also larger, which can be seen as an effect of stress triaxiality on twinning.

Fig. 20 shows the distribution of extension twin v.f. in the RN10 and RN2 specimens at two nominal strains, $\varepsilon_{\mathrm{A}}=0.1$ (Fig. 20a-20b) and $\varepsilon_{\mathrm{A}}=0.35$ (Fig. 20c-20d). The latter also indicates the locations of the regions that have undergone twin induced lattice reorientation. Relating the distribution of lateral stresses (Fig. 19) and the distribution of extension twin v.f. (Fig. 20), we observe that for a given stress triaxiality condition, regions of twinned volumes are not the regions with maximum tensile $\sigma_{[0001]}$ or the maximum compressive $\sigma_{[10 \overline{10}]}$ occur. Instead, twinning arises as a result of the complex interaction of the tensile and compressive lateral stresses along [0001] and [1010], respectively. Twinned regions are concentrated at the surface of notch root (along diametrical [0001]) in the RN2 specimen. In the RN10 specimens, the twinned regions are located at the surface of the notch above and below the notch root.

3. Contraction Twinning: Fig. 21 shows the distribution of contraction twin v.f. in the smooth, RN10 and RN2 specimens. Although strain has accrued on the contraction twin planes, the v.f. is much below the critical v.f. required for twin induced lattice reorientation. The low contraction twin v.f. in the simulations is because of the assumed values of CRSS and hardening parameters (there is lack of sufficient information on contraction twinning in literature). In addition, our model adopts a non-saturation hardening evolution for this mode, which makes it more difficult to sustain. Though the simulations do not predict the existence of reoriented regions, Kondori and Benzerga (2014a) suggest that contraction twinning is one of the two possible damage initiation sites. The role of contraction twins in initiating failure has been invoked in the past (Yoshinaga and Horiuchi, 1963; Barnett, 2007). It is noteworthy that the CRSS value used for contraction twinning of the alloys was assumed (due to lack of data from literature); in fact the CRSS and hardening parameters used in the simulations might be higher than the parameters corresponding to AZ31 leading to the absence of twin reoriented regions in the simulations. 
Since the deformation anisotropy depends on the activity of various deformation systems it is tempting to establish a relationship, if only approximate, between the two for loading along the rolling direction. In general, a simple relationship may not be possible because of the inhomogeneous deformation over the specimen volume. However, for local regions where deformations are homogeneous, we may define a ratio $\mathrm{R}_{\mathrm{L}}{ }^{\prime}=\frac{\dot{\varepsilon}_{[10 \overline{1} 0]}}{\dot{\varepsilon}_{[0001]}}$. If elastic strains are ignored, $\mathrm{R}_{\mathrm{L}}{ }^{\prime}$ becomes

$$
\mathrm{R}_{\mathrm{L}}^{\prime}=\frac{\dot{\varepsilon}_{[10 \overline{1} 0]}}{\dot{\varepsilon}_{[0001]}} \approx \frac{\left(\sum_{\alpha=1}^{N} \dot{\gamma}^{\alpha} \mathbf{P}^{\alpha}\right)_{[10 \overline{1} 0]}}{\left(\sum_{\alpha=1}^{N} \dot{\gamma}^{\alpha} \mathbf{P}^{\alpha}\right)_{[0001]}}
$$

where $N$ is the total number of deformation systems, $\mathbf{P}^{\alpha}$ is the symmetric part of the Schmid tensor. Note that a deformation system causing strain in [10̄0] may also cause strain in [0001] and vice-versa. For the case where there is there no such coupling the relationship becomes tractable; e.g. for the smooth specimen case

$$
\mathcal{Z}=\frac{\left(\sum_{\alpha=1}^{N} \zeta^{\alpha} \mathbf{P}^{\alpha}\right)_{[10 \overline{1} 0]}}{\left(\sum_{\alpha=1}^{N} \zeta^{\alpha} \mathbf{P}^{\alpha}\right)_{[0001]}}=\left(\frac{0.433 \zeta^{\operatorname{prism}\langle a\rangle}}{0.446 \zeta^{\operatorname{pyr}\langle c+a\rangle}+0.415 \zeta^{\mathrm{CT}}}\right)
$$

where $\zeta^{\alpha}=\dot{\gamma}^{\alpha} / \dot{\gamma}^{\text {total }}$ is the area averaged relative activity in the cross-sectional plane.

As shown in Fig. 22 there is a good correspondence between $R_{\mathrm{L}}{ }^{\prime}$ obtained directly from the simulations and $\mathcal{Z}$ (Eq. (18)) at all strains for alloy-2 and for small to moderate strains for pure $\mathrm{Mg}$. The deviation beyond moderate strains in the case of pure Mg is because elastic strains, which form a significant component of $\varepsilon_{[0001]}$ in pure $\mathrm{Mg}$, are ignored in calculating $\mathcal{Z}$ but are included in $R_{\mathrm{L}}^{\prime}$.

\section{Closing remarks}

In this work, the interplay between stress triaxiality and inherent plastic anisotropy was investigated in $\mathrm{Mg}$ single crystals using crystal plasticity finite element simulations. Details aside, the results indicate the following:

- For all the specimen geometries considered, the initial circular cross sections transforms into an oval upon deformation. This behavior is characteristic of anisotropic materials and is caused by unequal straining of the primary deformation systems in the specimen cross section. 
- The initial CRSS parameters play an important role in setting up the macroscopic deformation anisotropy. This leads to qualitative and quantitative differences between the pure $\mathrm{Mg}$ and alloy predictions. In addition to the activity of non-basal systems relative to basal slip, much of the macroscopic deformation anisotropy and its micromechanics depends on the contributions of non-basal systems relative to each other.

- In situations where alloying reduces the inherent plastic anisotropy (such as the cases considered here) the macroscopic deformation anisotropy in smooth specimens also decreases. In comparison, the behavior of such alloyed notched specimens is more subtle because of the emergence of lateral stresses that can trigger special deformation systems (e.g. extension twinning). This dependence of deformation mechanisms on the inherent plastic anisotropy is important because of its likely implications on the ductility and failure mode.

- The dependence of the deformation anisotropy on the plastic anisotropy can be well corroborated with changes in the underlying micromechanics. Since at the single crystal level the material anisotropy is a function of the alloying, modifying alloy composition to tune the anisotropy between the basal and non-basal slip and amongst the non-basal slip systems, is desirable in order to design for ductility under triaxial stress states.

- Triaxiality and plastic strain distributions in notched specimens corroborate qualitatively some observations of nascent or well developed cracks in various locations, notably close to the notch root and eventually away from the equator. Unlike most metallic materials where the locations of peak triaxiality and peak plastic strain are such that cracking initiates at the center, in $\mathrm{HCP}$ materials such as $\mathrm{Mg}$ the separation between triaxiality and plastic strain peaks is more complex.

- Textural variations in actual polycrystalline HCP microstructures most likely will mediate the effect of inherent (single crystal level) plastic anisotropy. The trends from the present work establish a basis for more detailed investigations and will serve as a benchmark to rationalize the deformation micromechanics of polycrystalline microstructures. A comprehensive investigation of plastic anisotropy, texture and triaxiality effects is key to developing micromechanics based damage models for HCP materials. 


\section{Acknowledgments}

BS acknowledges NUS Research Scholarship from Ministry of Education (MoE), Singapore. SPJ acknowledges partial financial support from MoE under AcRF Tier 1 Grant \# 265-000-459-112. AAB acknowledges partial support from the National Science Foundation (Grant Number DMR0844082).

\section{References}

Agnew, S., Duygulu, O., 2005. Plastic anisotropy and the role of non-basal slip in magnesium alloy AZ31B. International Journal of Plasticity 21, 1161-1193.

Agnew, S., Tomé, C., Brown, D., Holden, T., Vogel, S., 2003. Study of slip mechanisms in a magnesium alloy by neutron diffraction and modeling. Scripta Materialia 48, 1003-1008.

Akhtar, A., Teghtsoonian, E., 1969a. Solid solution strengthening of magnesium single crystals: I alloying behaviour in basal slip. Acta Metallurgica 17, 1339-1349.

Akhtar, A., Teghtsoonian, E., 1969b. Solid solution strengthening of magnesium single crystals: II the effect of solute on the ease of prismatic slip. Acta Metallurgica 17, 1351-1356.

Alves, M., Jones, N., 1999. Influence of hydrostatic stress on failure of axisymmetric notched specimens. Journal of the Mechanics and Physics of Solids 47, $643-667$.

Asaro, R.J., Needleman, A., 1985. Overview no. 42: Texture development and strain hardening in rate dependent polycrystals. Acta Metallurgica 33, 923-953.

Barnett, M., 2007. Twinning and the ductility of magnesium alloys: Part II: Contraction twins. Materials Science and Engineering: A 464, 8-16.

Benzerga, A., 2000. Rupture ductile des tôles anisotropes. Simulation de la propagation longitudinale dans un tube pressurisé. Ph.D. thesis. Ecole Nationale Supérieure des Mines de Paris.

Benzerga, A., Besson, J., Pineau, A., 2004a. Anisotropic ductile fracture: Part I: experiments. Acta Materialia 52, 4623-4638. 
Benzerga, A., Besson, J., Pineau, A., 2004b. Anisotropic ductile fracture: Part II: theory. Acta Materialia 52, 4639-4650.

Benzerga, A., Leblond, J.B., 2010. Ductile fracture by void growth to coalescence. Advances in Applied Mechanics 44, 169-305.

Benzerga, A.A., Besson, J., 2001. Plastic potentials for anisotropic porous solids. European Journal of Mechanics-A/Solids 20, 397-434.

Benzerga, A.A., Besson, J., Pineau, A., 1999. Coalescence-Controlled Anisotropic Ductile Fracture $121,221-229$.

Benzerga, A.A., Leblond, J.B., Needleman, A., Tvergaard, V., 2016. Ductile Failure Modeling. International Journal of Fracture To be submitted.

Bohlen, J., Nürnberg, M.R., Senn, J.W., Letzig, D., Agnew, S.R., 2007. The texture and anisotropy of magnesium-zinc-rare earth alloy sheets. Acta Materialia 55, 2101-2112.

Chapuis, A., Driver, J.H., 2011. Temperature dependency of slip and twinning in plane strain compressed magnesium single crystals. Acta Materialia 59, 1986-1994.

Christian, J.W., Mahajan, S., 1995. Deformation twinning. Progress in Materials Science 39, 1-157.

Gurson, A., 1977. Continuum theory of ductile rupture by void nucleation and growth: Part I: Yield criteria and flow rules for porous ductile media. Journal of engineering materials and technology $99,2-15$.

Han, X., Besson, J., Forest, S., Tanguy, B., Bugat, S., 2013. A yield function for single crystals containing voids. International Journal of Solids and Structures 50, $2115-2131$.

Hancock, J., Brown, D., 1983. On the role of strain and stress state in ductile failure. Journal of the Mechanics and Physics of Solids 31, 1-24.

Hancock, J.W., MacKenzie, A.C., 1976. On the mechanisms of ductile failure in high-strength steels subjected to multi-axial stress states. Journal of the Mechanics and Physics of Solids 24, $147-169$. 
Hong, S.G., Park, S.H., Lee, C.S., 2010. Role of $\{10 \overline{1} 2\}$ twinning characteristics in the deformation behavior of a polycrystalline magnesium alloy. Acta Materialia 58, 5873-5885.

Huang, Y., 1991. A User-material Subroutine Incroporating Single Crystal Plasticity in the ABAQUS Finite Element Program. Harvard Univ.

Hutchinson, W., Barnett, M., 2010. Effective values of critical resolved shear stress for slip in polycrystalline magnesium and other hcp metals. Scripta Materialia 63, 737-740.

Kalidindi, S.R., 1998. Incorporation of deformation twinning in crystal plasticity models. Journal of the Mechanics and Physics of Solids 46, 267-290.

Kaushik, V., Narasimhan, R., Mishra, R., 2014. Experimental study of fracture behavior of magnesium single crystals. Materials Science and Engineering: A 590, 174-185.

Kelley, E., 1967. The plastic deformation of magnesium. Technical Report. DTIC Document.

Keralavarma, S., Benzerga, A., 2010. A constitutive model for plastically anisotropic solids with non-spherical voids. Journal of the Mechanics and Physics of Solids 58, 874-901.

Keralavarma, S.M., Benzerga, A.A., 2008. An approximate yield criterion for anisotropic porous media. Comptes Rendus Mécanique 336, 685-692.

Keralavarma, S.M., Hoelscher, S., Benzerga, A.A., 2011. Void growth and coalescence in anisotropic plastic solids. International Journal of Solids and Structures 48, 1696-1710.

Kondori, B., 2015. Ductile fracture of Magnesium alloys: characterization and modeling. Ph.D. thesis. Texas A\&M University, USA.

Kondori, B., Benzerga, A., 2014a. Effect of stress triaxiality on the flow and fracture of Mg alloy AZ31. Metallurgical and Materials Transactions A 45, 3292-3307.

Kondori, B., Benzerga, A., 2014b. Fracture strains, damage mechanisms and anisotropy in a magnesium alloy across a range of stress triaxialities. Experimental Mechanics 54, 493-499.

Lebensohn, R.A., Tomé, C., 1993. A self-consistent anisotropic approach for the simulation of plastic deformation and texture development of polycrystals: application to zirconium alloys. Acta Metallurgica et Materialia 41, 2611-2624. 
Lou, X., Li, M., Boger, R., Agnew, S., Wagoner, R., 2007. Hardening evolution of AZ31B mg sheet. International Journal of Plasticity 23, 44-86.

Mbiakop, A., Constantinescu, A., Danas, K., 2015. An analytical model for porous single crystals with ellipsoidal voids. Journal of the Mechanics and Physics of Solids 84, 436-467.

Monchiet, V., Cazacu, O., Charkaluk, E., Kondo, D., 2008. Macroscopic yield criteria for plastic anisotropic materials containing spheroidal voids. International Journal of Plasticity 24, 11581189.

Needleman, A., Tvergaard, V., 1984. An analysis of ductile rupture in notched bars. Journal of the Mechanics and Physics of Solids 32, 461-490.

Paux, J., Morin, L., Brenner, R., Kondo, D., 2015. An approximate yield criterion for porous single crystals. European Journal of Mechanics - A/Solids 51, 1 - 10.

Pineau, A., Benzerga, A.A., Pardoen, T., 2016. Overview article: Failure of Metals I. Brittle and Ductile Fracture. Acta Materialia To Appear.

Raeisinia, B., Agnew, S., Akhtar, A., 2011. Incorporation of solid solution alloying effects into polycrystal modeling of Mg alloys. Metallurgical and Materials Transactions A 42, 1418-1430.

Rice, J., Tracey, D., 1969. On the ductile enlargement of voids in triaxial stress fields. Journal of the Mechanics and Physics of Solids 17, 201-217.

Selvarajou, B., Shin, J., Ha, T., Choi, I., Joshi, S., Han, H., 2014. Orientation-dependent indentation response of magnesium single crystals: Modeling and experiments. Acta Materialia 81, $358-376$.

Shin, J., Kim, S., Ha, T., Oh, K., Choi, I., Han, H., 2013. Nanoindentation study for deformation twinning of magnesium single crystal. Scripta Materialia 68, 483-486.

Slutsky, L., Garland, C., 1957. Elastic constants of magnesium from $4.2 \mathrm{~K}$ to $300 \mathrm{~K}$. Physical Review 107, 972.

Staroselsky, A., Anand, L., 1998. Inelastic deformation of polycrystalline face centered cubic materials by slip and twinning. Journal of the Mechanics and Physics of Solids 46, 671-696. 
Staroselsky, A., Anand, L., 2003. A constitutive model for hcp materials deforming by slip and twinning: application to magnesium alloy AZ31B. International journal of Plasticity 19, 18431864.

Stewart, J.B., Cazacu, O., 2011. Analytical yield criterion for an anisotropic material containing spherical voids and exhibiting tension-compression asymmetry. International Journal of Solids and Structures 48, 357-373.

Systèmes, D., 2012. Abaqus 6.12 theory manual. Dassault Systèmes Simulia Corp., Providence, Rhode Island .

Tanguy, B., Luu, T.T., Perrin, G., Pineau, A., Besson, J., 2008. Plastic and damage behaviour of a high strength X100 pipeline steel: Experiments and modelling. International Journal of Pressure Vessels and Piping 85, 322-335.

Tomé, C.N., Lebensohn, R.A., Kocks, U.F., 1991. A model for texture development dominated by deformation twinning: application to zirconium alloys. Acta Metallurgica et Materialia 39, $2667-2680$.

Wenk, H.R., Van Houtte, P., 2004. Texture and anisotropy. Reports on Progress in Physics 67, 1367.

Wu, X., Kalidindi, S.R., Necker, C., Salem, A.A., 2008. Modeling anisotropic stress-strain response and crystallographic texture evolution in $\alpha$-titanium during large plastic deformation using taylor-type models: influence of initial texture and purity. Metallurgical and Materials Transactions A 39, 3046-3054.

Yerra, S., Tekog, C., Scheyvaerts, F., Delannay, L., Van Houtte, P., Pardoen, T., et al., 2010. Void growth and coalescence in single crystals. International journal of solids and structures 47, $1016-1029$.

Yoo, M., 1981. Slip, twinning, and fracture in hexagonal close-packed metals. Metallurgical Transactions A 12, 409-418. 
Yoshinaga, H., Horiuchi, R., 1963. Deformation mechanisms in magnesium single crystals compressed in the direction parallel to hexagonal axis. Transactions of the Japan Institute of Metals $4,1-8$.

Zhang, J., Joshi, S., 2012. Phenomenological crystal plasticity modeling and detailed micromechanical investigations of pure magnesium. Journal of the Mechanics and Physics of Solids 60, 945-972. 


\section{Tables}

Table 1 Material parameters for pure Mg

\begin{tabular}{|c|c|c|c|c|}
\hline & $\tau_{0}(\mathrm{MPa})$ & $h_{\circ}(\mathrm{MPa})$ & $\tau_{s}(\mathrm{MPa})$ & \\
\hline Basal Slip & 0.5 & 20 & - & \\
\hline Prismatic $\langle a\rangle$ Slip & 25 & 1500 & 85 & \\
\hline Pyramidal $\langle a\rangle$ Slip & 25 & 1500 & 85 & \\
\hline \multirow[t]{2}{*}{ Pyramidal $\langle c+a\rangle$ Slip } & 40 & 3000 & 150 & \\
\hline & $\tau_{0}(\mathrm{MPa})$ & $h_{e t}(\mathrm{MPa})$ & $\tau_{s_{-} e t}(\mathrm{MPa})$ & $h_{e t \_s l}(\mathrm{MPa})$ \\
\hline \multirow[t]{2}{*}{ Extension Twinning } & 3.5 & 100 & 20 & 100 \\
\hline & $\tau_{0}(\mathrm{MPa})$ & $H_{c t}(\mathrm{MPa})$ & $H_{c t \_s l}(\mathrm{MPa})$ & $b$ \\
\hline Contraction Twinning & 55 & 6000 & 15 & 0.05 \\
\hline
\end{tabular}

Table 2 Comparison of material parameters of Pure $\mathrm{Mg}$ (high plastic anisotropy), alloy-1 (intermediate plastic anisotropy) and alloy-2 (low plastic anisotropy

\begin{tabular}{cccccccccc}
\hline & \multicolumn{2}{c}{ Pure $\mathrm{Mg}$} & & \multicolumn{2}{c}{ alloy-1 } & & \multicolumn{2}{c}{ alloy-2 } \\
\cline { 2 - 3 } \cline { 8 - 9 } Slip/twin system & $\tau_{0}(\mathrm{MPa})$ & $\frac{\tau_{0}}{\tau_{0}^{\text {prism }}}$ & & $\tau_{0}(\mathrm{MPa})$ & $\frac{\tau_{0}}{\tau_{0}^{\text {prism }}}$ & & $\tau_{0}(\mathrm{MPa})$ & $\frac{\tau_{0}}{\tau_{0}^{\text {prism }}}$ \\
\hline Basal Slip & 0.5 & 0.02 & & $10^{\#}$ & 0.18 & & $10^{\#}$ & 0.18 \\
Prismatic $\langle a\rangle$ Slip & 25 & 1 & & $55^{\#}$ & 1 & & $55^{\#}$ & 1 \\
Pyramidal $\langle a\rangle$ Slip & 25 & 1 & & $55^{\#}$ & 1 & & $55^{\#}$ & 1 \\
Pyramidal $\langle c+a\rangle$ Slip & 40 & 1.6 & & $70^{\#}$ & 1.3 & & $60^{\#}$ & 1.1 \\
Extension Twinning & 3.5 & 0.14 & & $15^{\#}$ & 0.27 & & $15^{\#}$ & 0.27 \\
Contraction Twinning & 55 & 1.4 & & $85^{*}$ & 1.55 & & $85^{*}$ & 1.55 \\
\hline
\end{tabular}

\# - (Agnew et al., 2003; Lou et al., 2007; Hong et al., 2010; Hutchinson and Barnett, 2010)

* - assumed 


\section{Figure Captions}

Figure 1. Comparison between the outline and location of macroscopic cracks in (a) a low carbon medium alloy steel (Benzerga, 2000); and (b) AZ31 magnesium alloy (Kondori, 2015).

Figure 2. Geometry and finite element meshes for the analyzed smooth and notched specimens.

Figure 3. Normalized load vs. normalized diametric reduction in the smooth and notched specimens of pure Mg single crystal. The open symbol in the smooth specimen indicates the initiation of strain localization.

Figure 4. Evolution of deformation anisotropy ratio $\left(R_{L}\right)$ as function of areal strain $\varepsilon_{A}$ in smooth and notched pure Mg specimens.

Figure 5. Dependence of $R_{L}$ on triaxiality for pure $M g$ corresponding to $\varepsilon_{A}=0.1$. The shaded ovals indicate deformed cross-sectional shapes and the light outline indicates original circular crosssection. The deformation is scaled up by a factor of 5 .

Figure 6. Distribution of triaxiality in the (a) RN10 and (b) RN2 specimens of pure Mg single crystal at $\varepsilon_{\mathrm{A}}=0.1$. (c) shows the triaxiality along [0001] and [1010] in the minimum section.

Figure 7. Evolution of relative activity in (a) Smooth, (b) RN10 and (c) RN2 specimens of pure Mg single crystal.

Figure 8. Distribution of lateral normal stresses $\sigma_{[0001]}$ and $\sigma_{[10 \overline{1} 0]}$ in RN10 (a-b) and RN2 (c-d) specimens of pure $\mathrm{Mg}$ single crystal at $\varepsilon_{\mathrm{A}}=0.1$

Figure 9. Plastic strain distributions in the (a) Smooth, (b) RN10 and (c) RN2 specimens of pure $\mathrm{Mg}$ single crystal at $\varepsilon_{\mathrm{A}}=0.1$. Strain localization along [1010] in the mid section of the smooth specimen is seen in panel (a).

Figure 10. Variation of equivalent plastic strain along [0001] and [1010] in the minimum section of the pure $\mathrm{Mg}$ notch specimens.

Figure 11. Prismatic $\langle a\rangle$ slip in the (a) Smooth, (b) RN10 and (c) RN2 specimens of pure Mg single crystal.

Figure 12. Basal slip in the (a) RN10 and (b) RN2 specimens of pure Mg single crystal. Basal Slip is inactive in the smooth specimen since the Schmid factor is zero. 
Figure 13. Pyramidal $\langle c+a\rangle$ slip in the (a) RN10 and (b) RN2 specimens of pure Mg single crystal. Though the Schmid factor for pyramidal $\langle c+a\rangle$ slip in the smooth specimen is high, it is inactive due to latent hardening.

Figure 14. Comparison of diametric reduction at fracture initiation strain in the pure $\mathrm{Mg}$, alloy-1 and alloy-2 specimens with the experiments as function of triaxiality.

Figure 15. Comparison of load obtained from the simulations in the pure $\mathrm{Mg}$, alloy-1 and alloy-2 specimens with the limit load in the experiments as function of triaxiality.

Figure 16. (a) Evolution of deformation anisotropy in the alloy-1 and alloy-2 specimens with overall areal strain, and (b) Comparison of $\mathrm{R}_{\mathrm{L}}$ in the pure, alloy-1 and alloy- 2 specimens with the experiments. The experimental $R_{L}$ values correspond to the fracture initiation strain and the same strain is used to extract $R_{L}$ from the simulations. The dashed line in (b) represents $R_{L}$ for an isotropic material.

Figure 17. Triaxiality along [0001] and [10̄0] in the minimum section of alloy-2 single crystal

Figure 18. Evolution of relative activity in (a) Smooth, (b) RN10 and (c) RN2 specimens of the alloy-2 single crystal.

Figure 19. (a) Lateral normal stresses that promote extension twinning in the notch region. Panels (b-e) show the distribution of lateral stresses $\sigma_{[0001]}$ and $\sigma_{[10 \overline{10}]}$ in RN10 (b-c) and RN2 (d-e) specimens of alloy-2 single crystal at $\varepsilon_{\mathrm{A}}=0.1$

Figure 20. Extension twin v.f. at $\varepsilon_{\mathrm{A}}=0.1$ in (a) RN10 (b) RN2, at $\varepsilon_{\mathrm{A}}=0.35$ in (c) RN10 (d) RN2 specimens of alloy-2 single crystal

Figure 21. Contraction twin v.f. in (a) Smooth, (b) RN10 (c) RN2 specimens of alloy-2 single crystal. $f^{\mathrm{CT}}<<f_{c}$ even at high values of strain.

Figure 22. Comparison of $\mathrm{R}_{\mathrm{L}}^{\prime}$ obtained from the simulations and $\mathcal{Z}$ calculated using the relative activity values. 


\section{Figures}

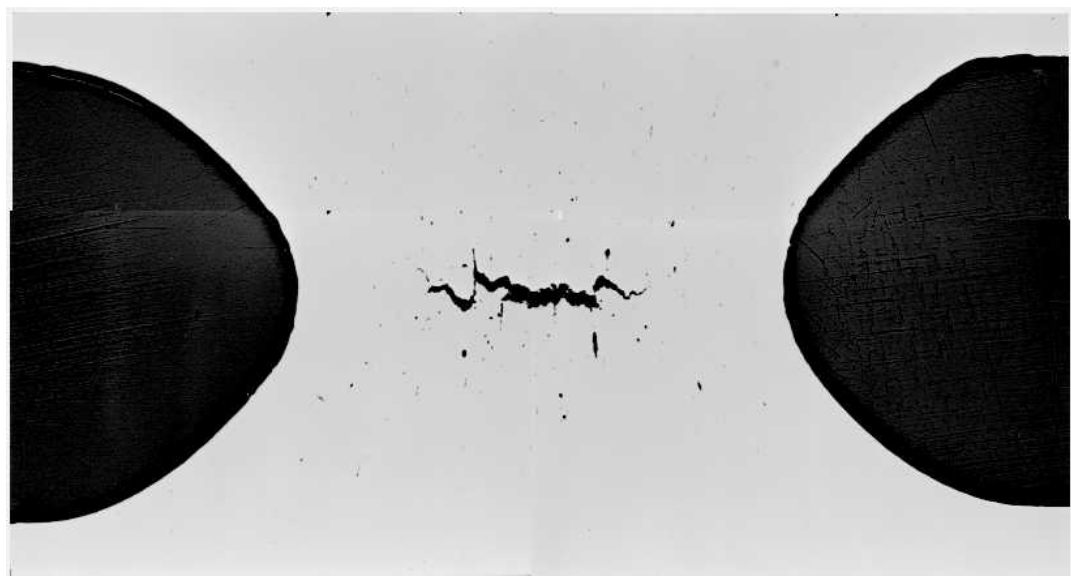

(a)

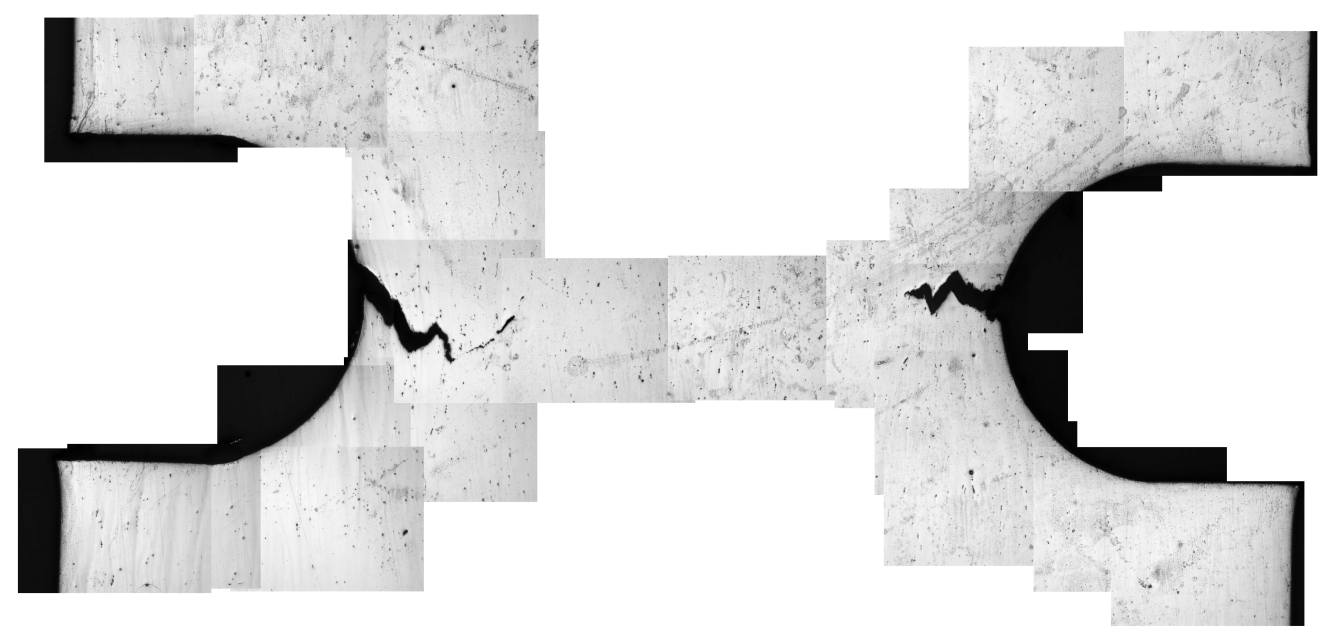

(b)

Figure 1 Comparison between the outline and location of macroscopic cracks in (a) a low carbon medium alloy steel (Benzerga, 2000); and (b) AZ31 magnesium alloy (Kondori, 2015). 


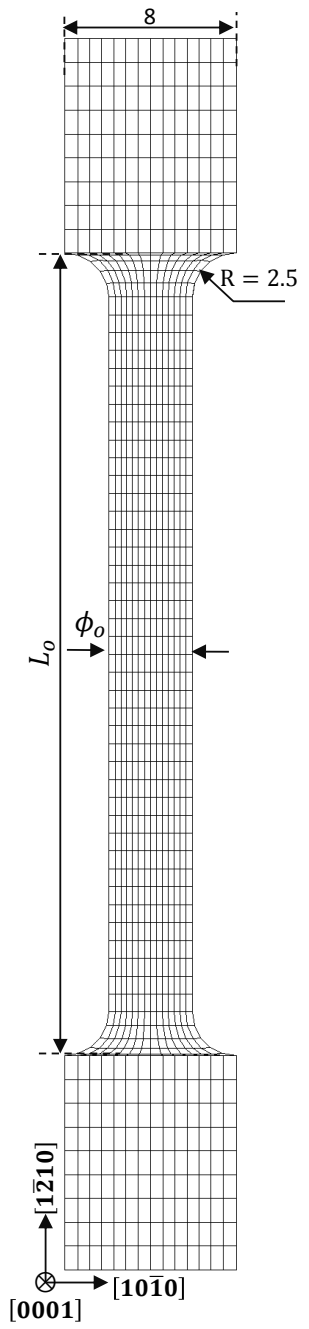

(a) Smooth

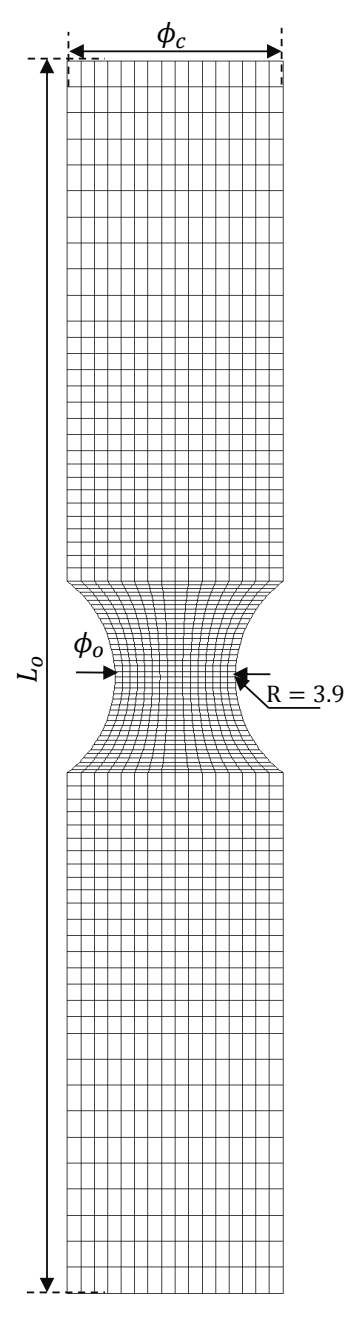

(b) RN10

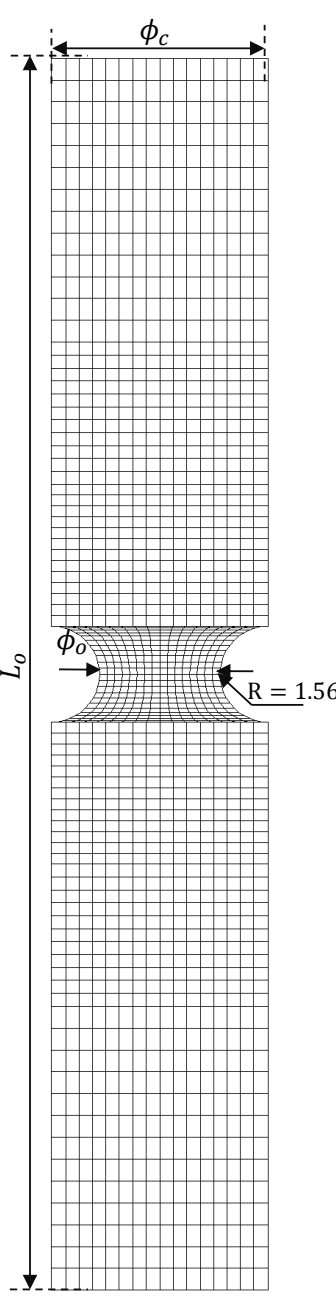

(c) RN4

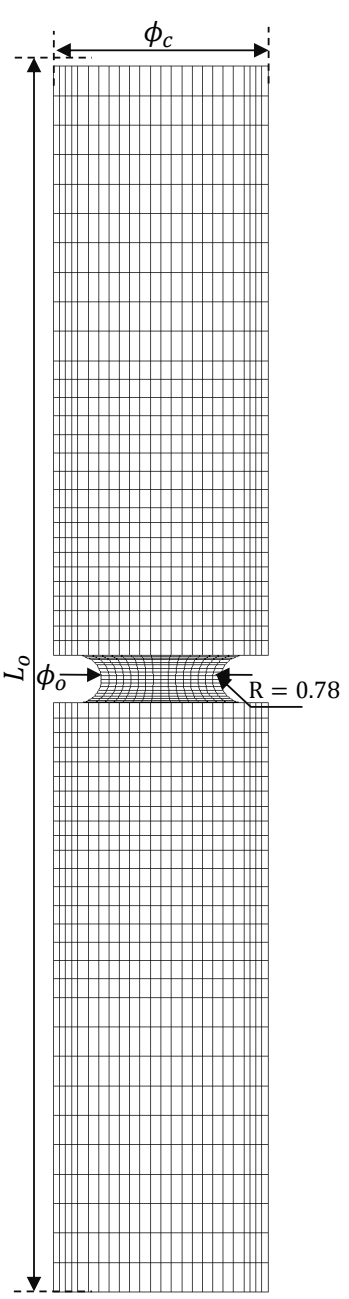

(d) RN2

Figure 2 Geometry and finite element meshes for the analyzed smooth and notched specimens. 


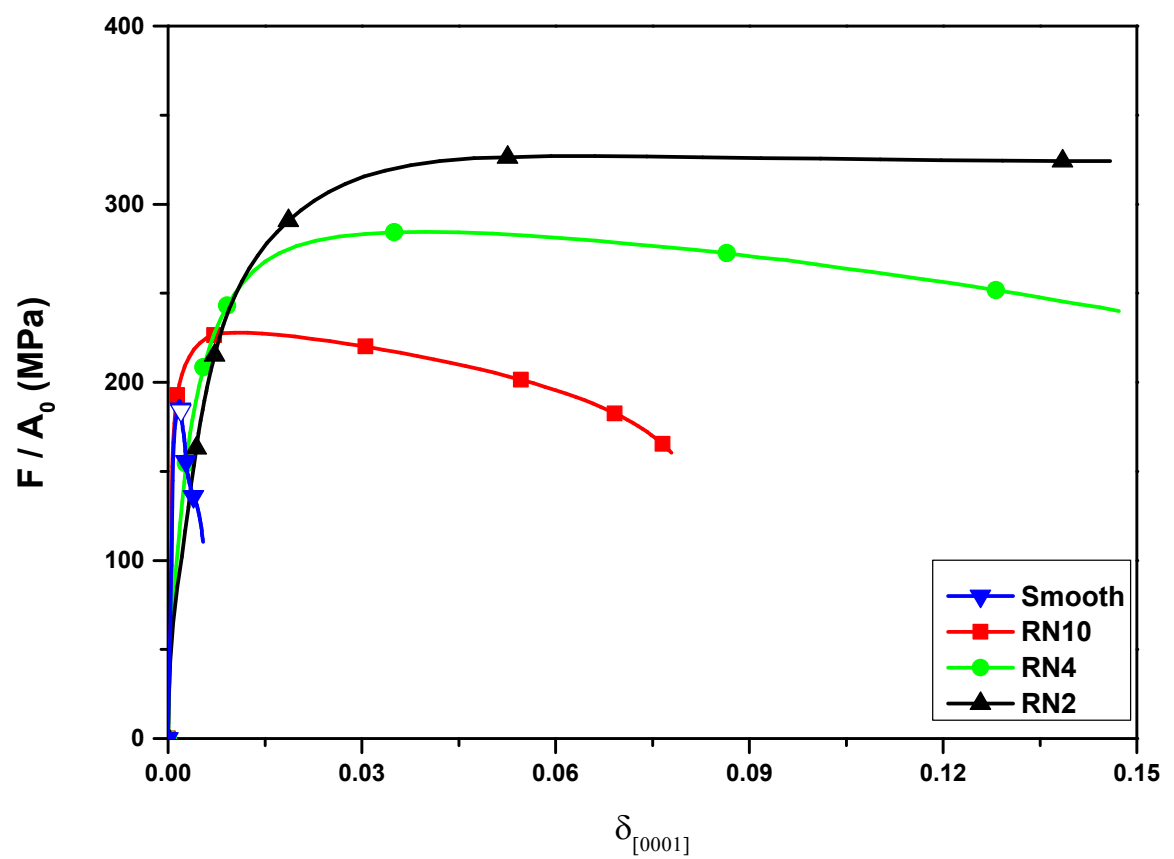

(a)

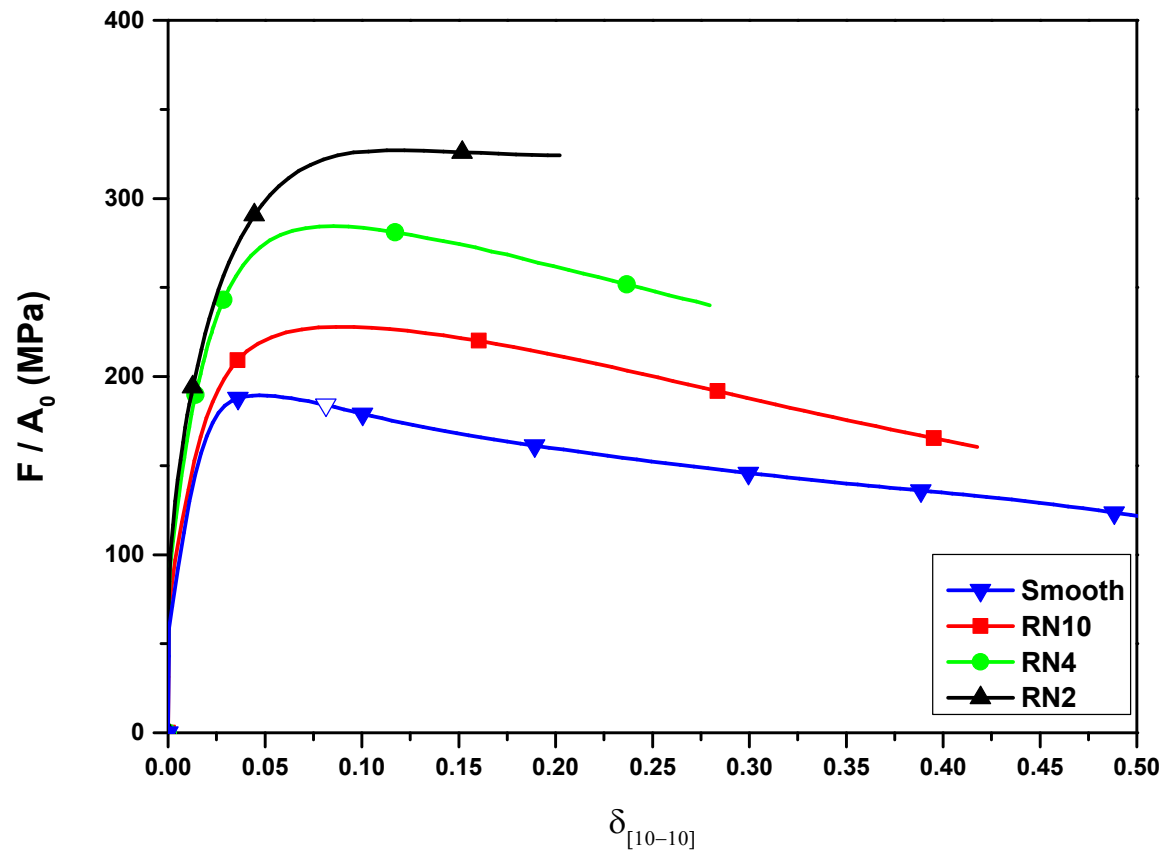

(b)

Figure 3 Normalized load vs. normalized diametric reduction in the smooth and notched specimens of pure $\mathrm{Mg}$ single crystal. The open symbol in the smooth specimen indicates the initiation of strain localization. 


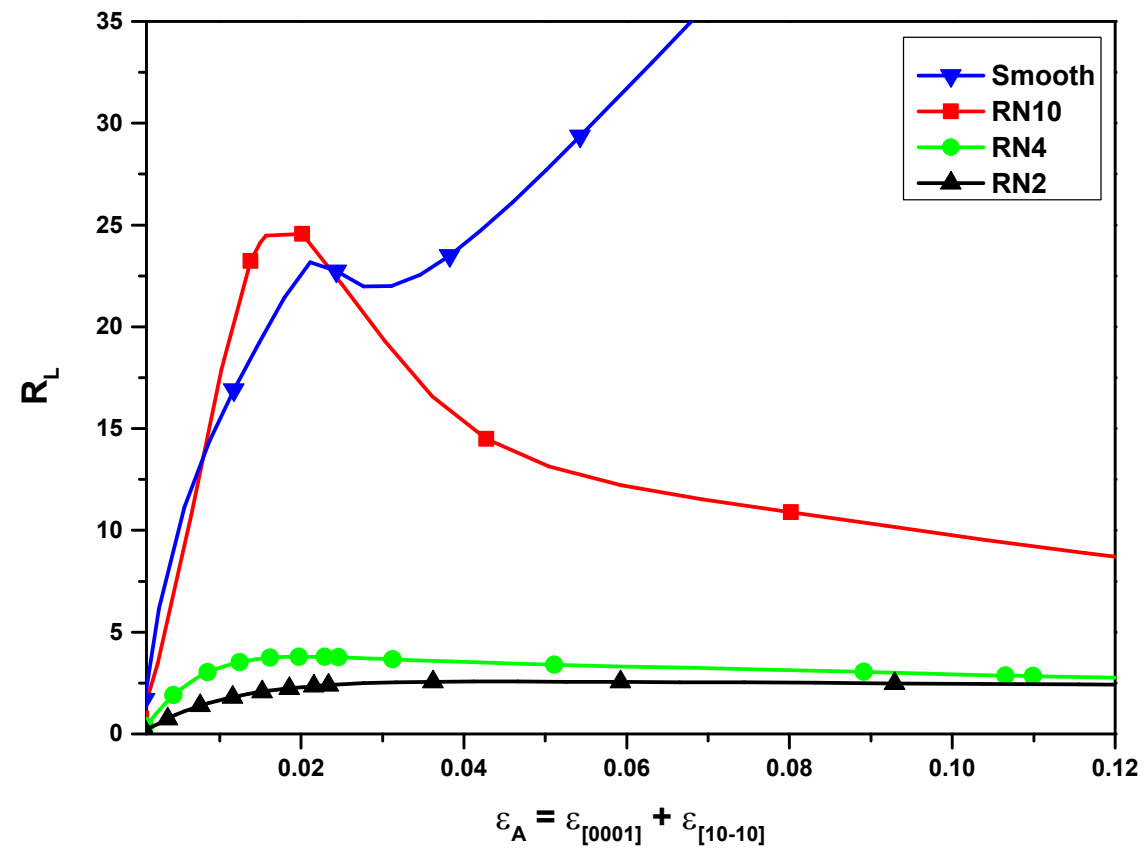

Figure 4 Evolution of deformation anisotropy ratio $\left(\mathrm{R}_{\mathrm{L}}\right)$ as function of areal strain $\varepsilon_{\mathrm{A}}$ in smooth and notched pure Mg specimens.

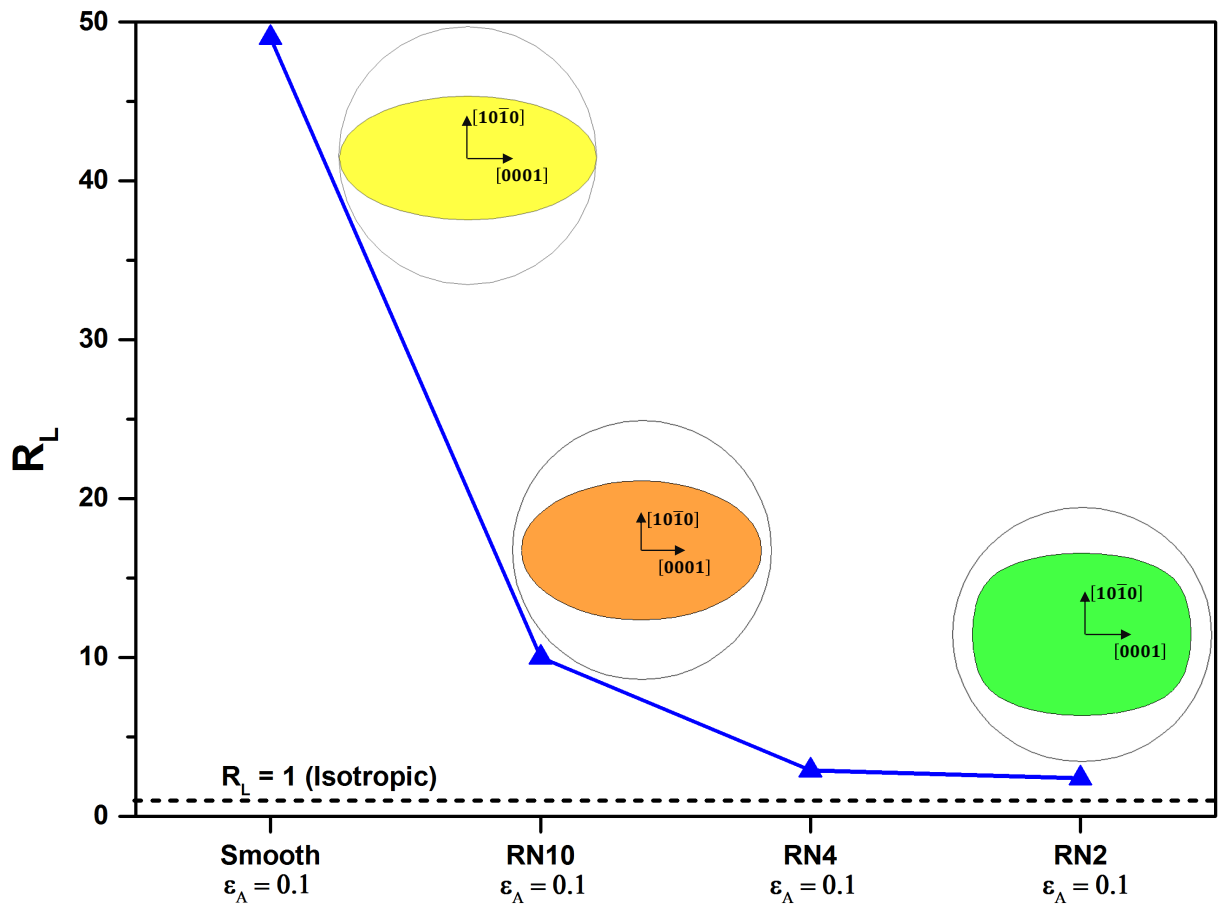

Figure 5 Dependence of $R_{\mathrm{L}}$ on triaxiality for pure $\mathrm{Mg}$ corresponding to $\varepsilon_{\mathrm{A}}=0.1$. The shaded ovals indicate deformed cross-sectional shapes and the light outline indicates original circular cross-section. The deformation is scaled up by a factor of 5 . 


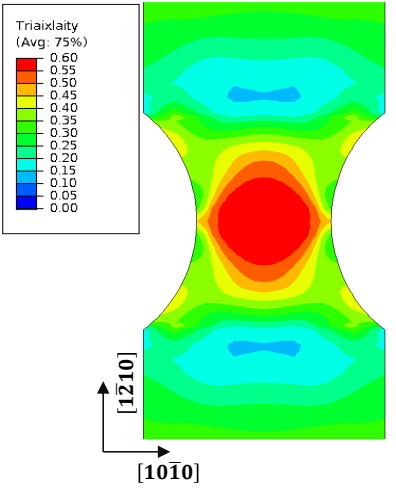

(a) RN10
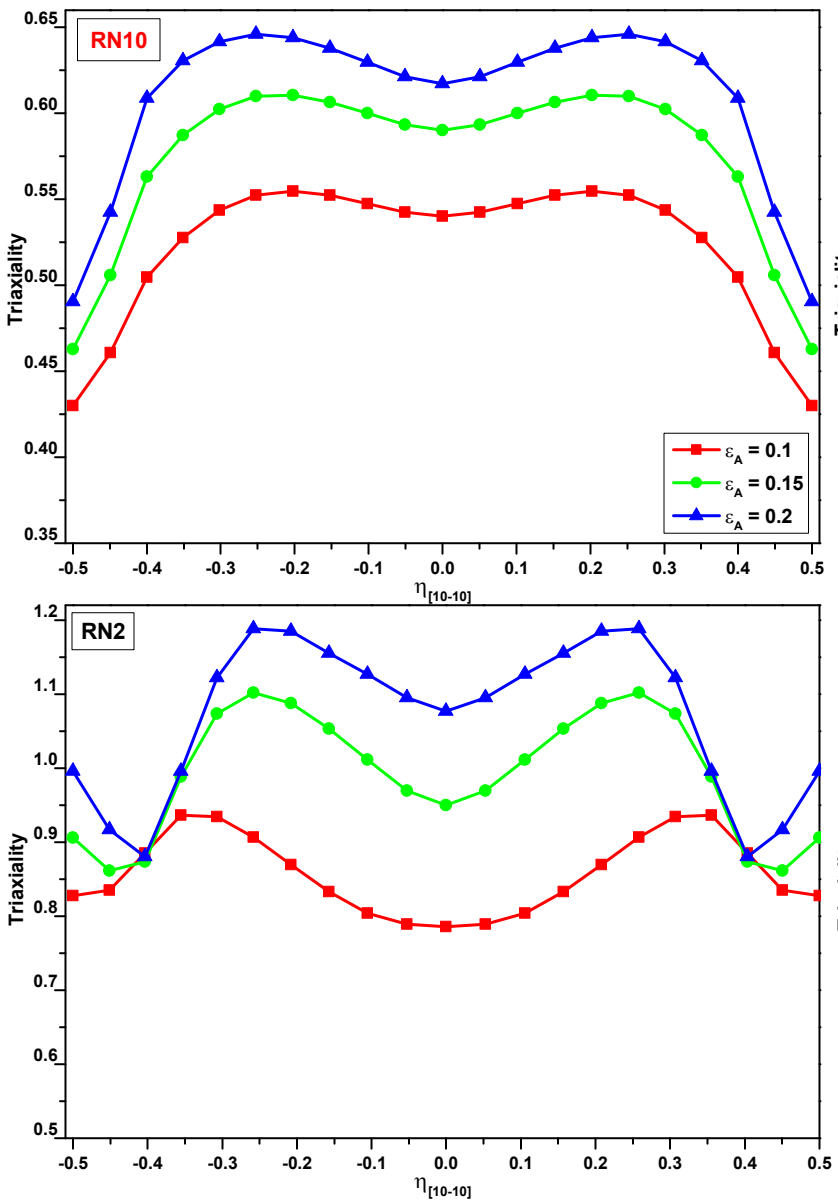

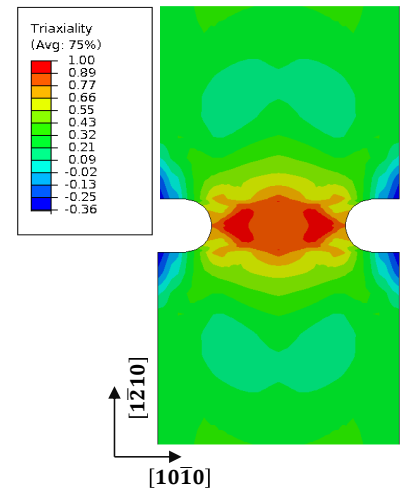

(b) RN2
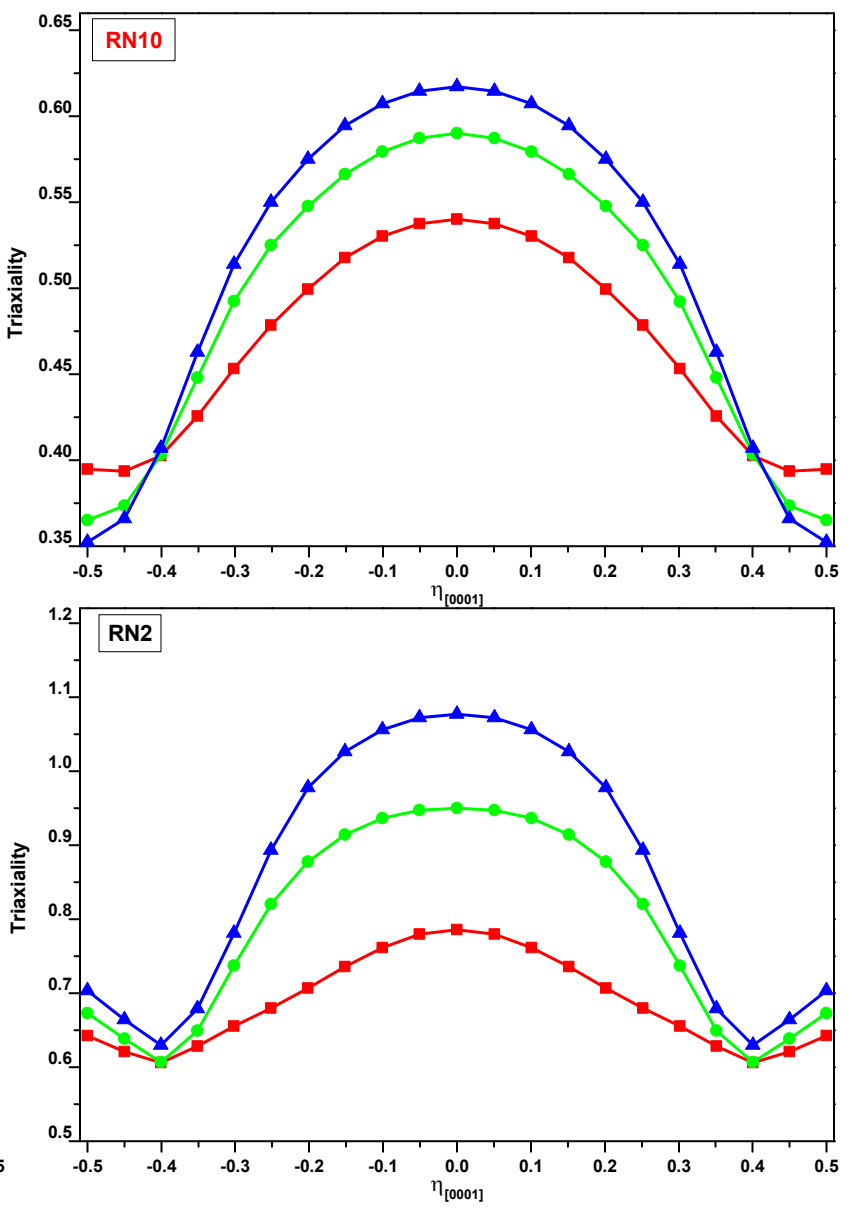

(c)

Figure 6 Distribution of triaxiality in the (a) RN10 and (b) RN2 specimens of pure Mg single crystal at $\varepsilon_{\mathrm{A}}=0.1$. (c) shows the triaxiality along [0001] and [1010] in the minimum section. 


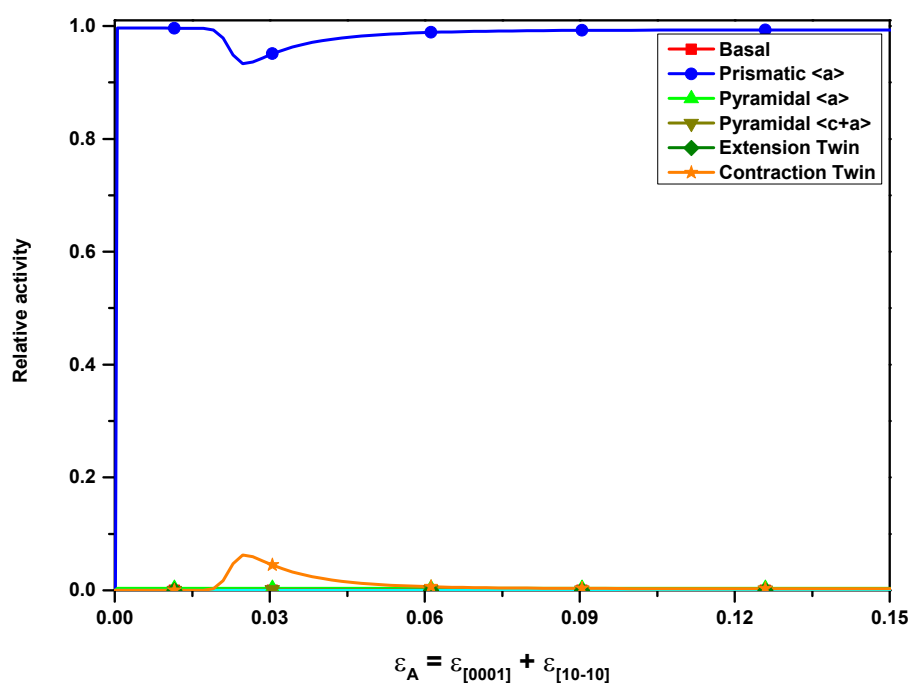

(a) Smooth

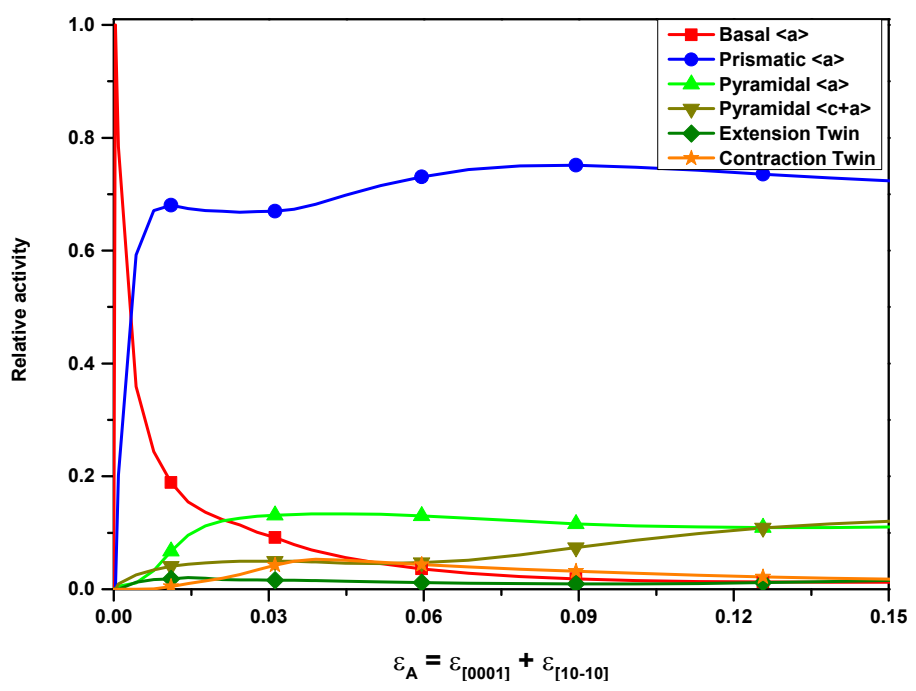

(b) RN10

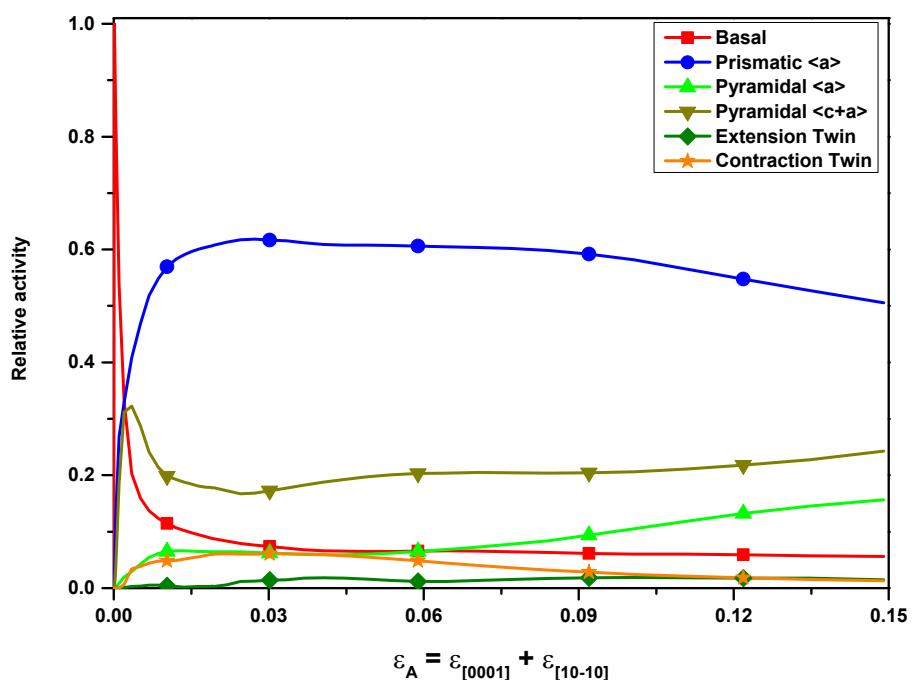

(c) $37 \mathrm{RN} 2$

Figure 7 Evolution of relative activity in (a) Smooth, (b) RN10 and (c) RN2 specimens of pure Mg single crystal. 


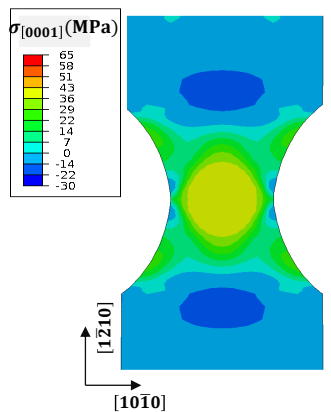

(a) $\sigma_{[0001]}$

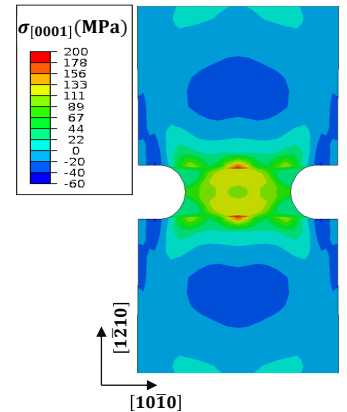

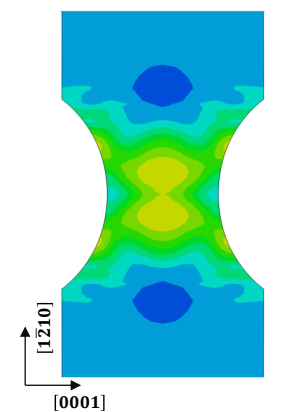

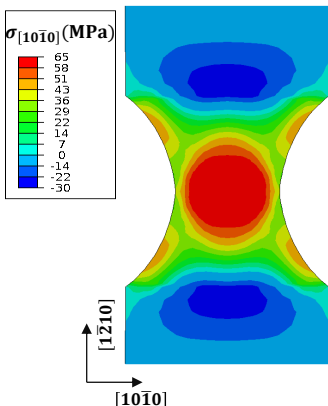

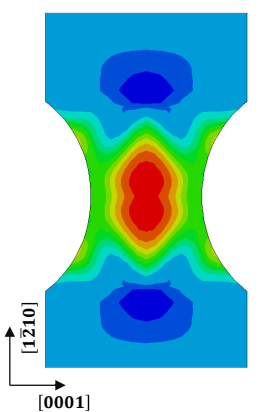

(b) $\sigma_{[10 \overline{1} 0]}$
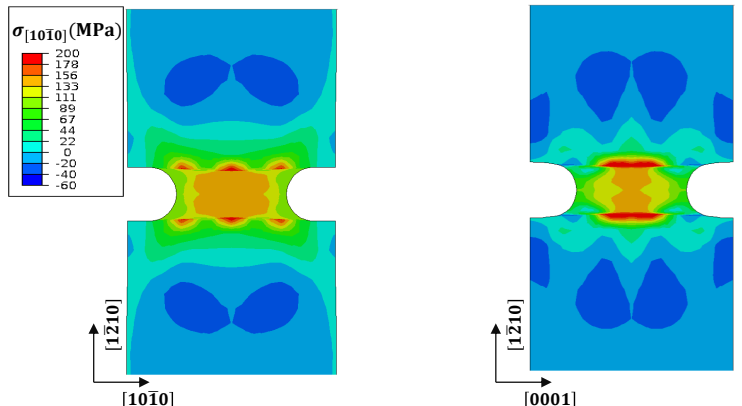

(d) $\sigma_{[10 \overline{1} 0]}$

Figure 8 Distribution of lateral normal stresses $\sigma_{[0001]}$ and $\sigma_{[10 \overline{1} 0]}$ in RN10 (a-b) and RN2 (c-d) specimens of pure $\mathrm{Mg}$ single crystal at $\varepsilon_{\mathrm{A}}=0.1$ 

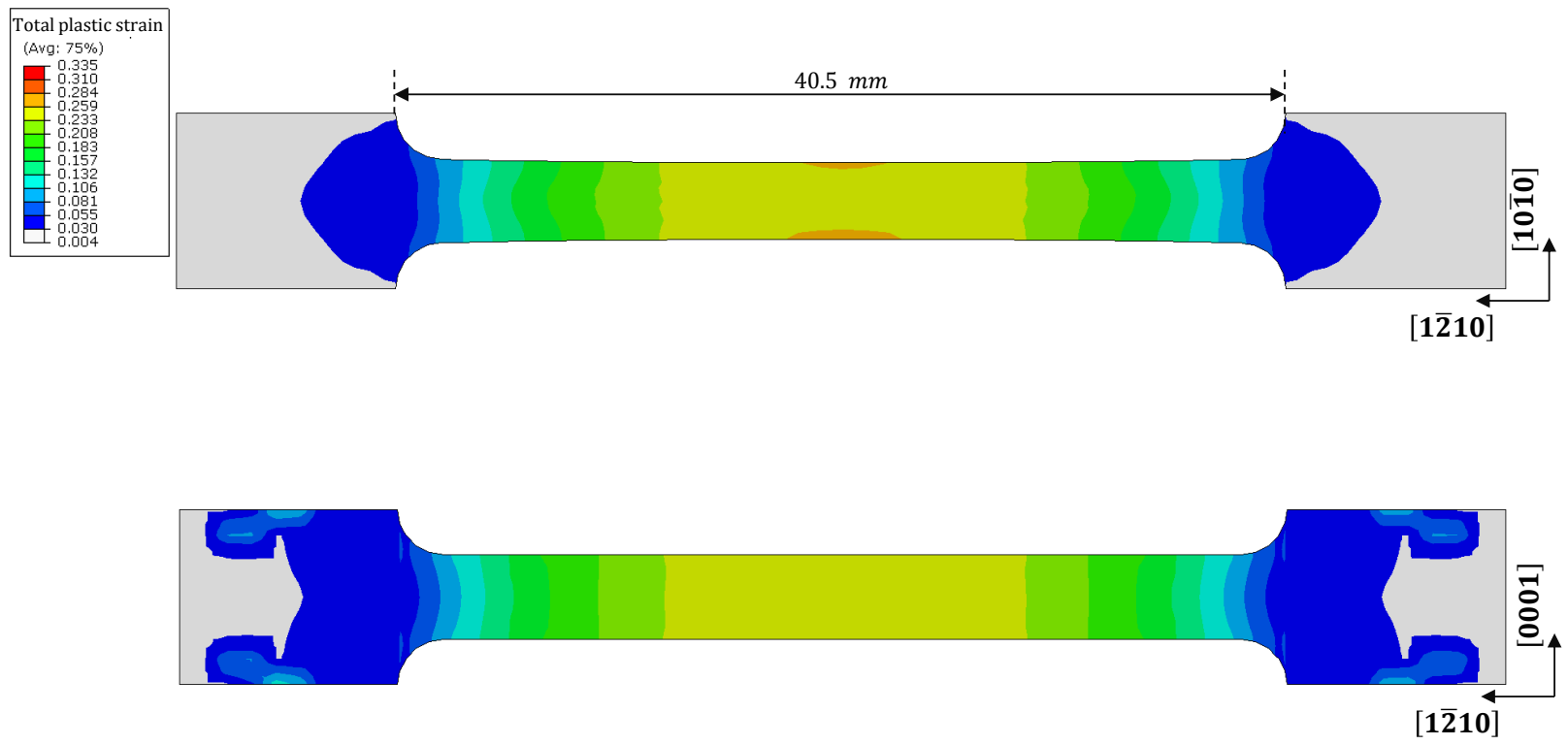

(a) Smooth

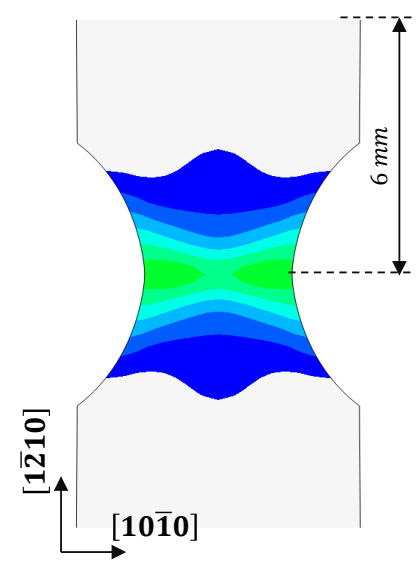

(b) RN10
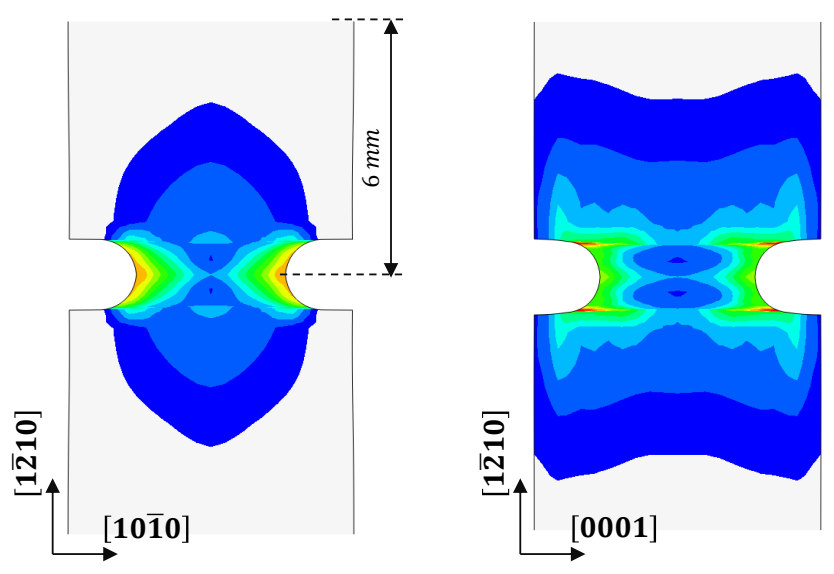

(c) RN2

Figure 9 Plastic strain distributions in the (a) Smooth, (b) RN10 and (c) RN2 specimens of pure Mg single crystal at $\varepsilon_{\mathrm{A}}=0.1$. Strain localization along [1010] in the mid section of the smooth specimen is seen in panel (a). 

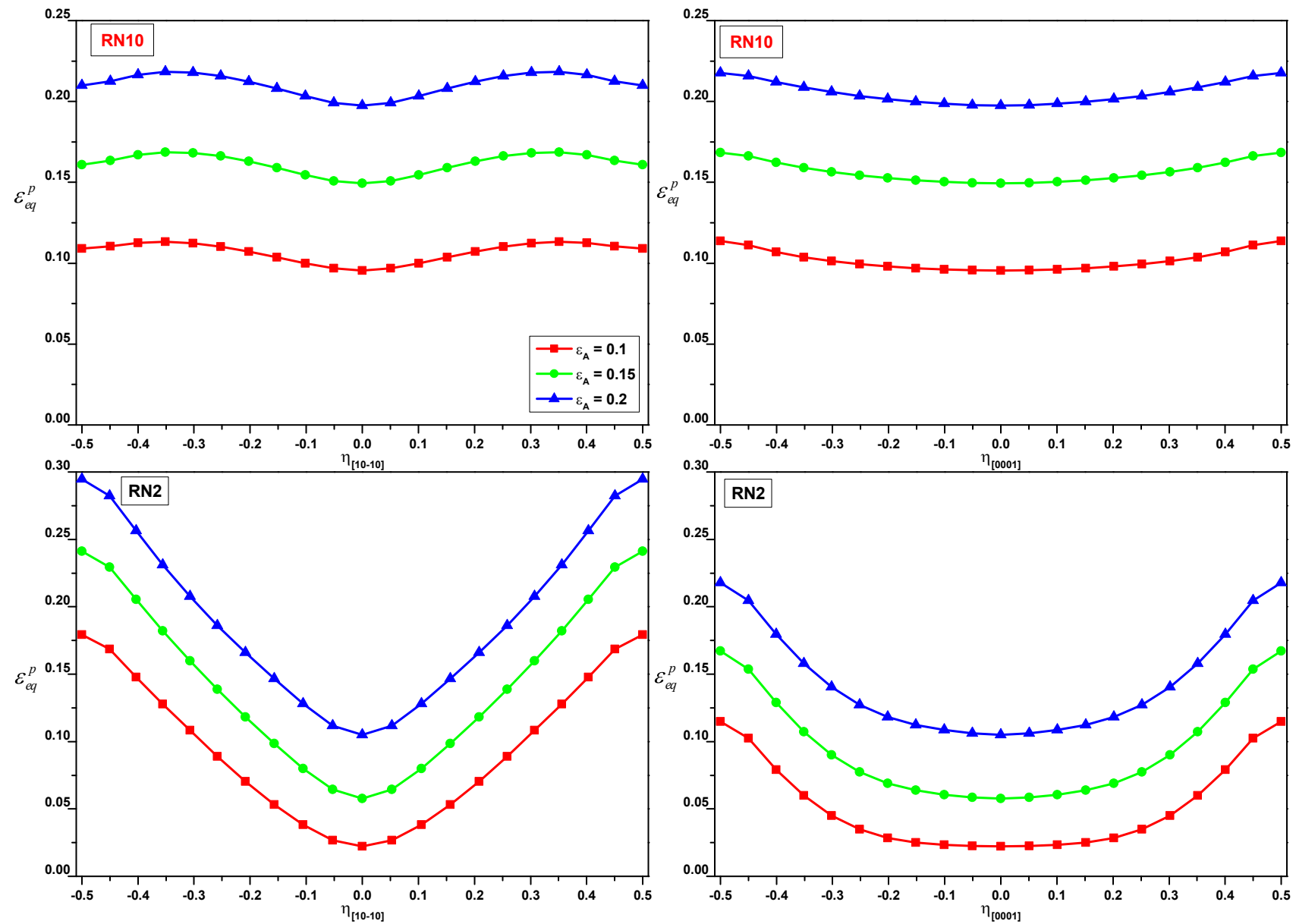

Figure 10 Variation of equivalent plastic strain along [0001] and [1010] in the minimum section of the pure $\mathrm{Mg}$ notch specimens. 

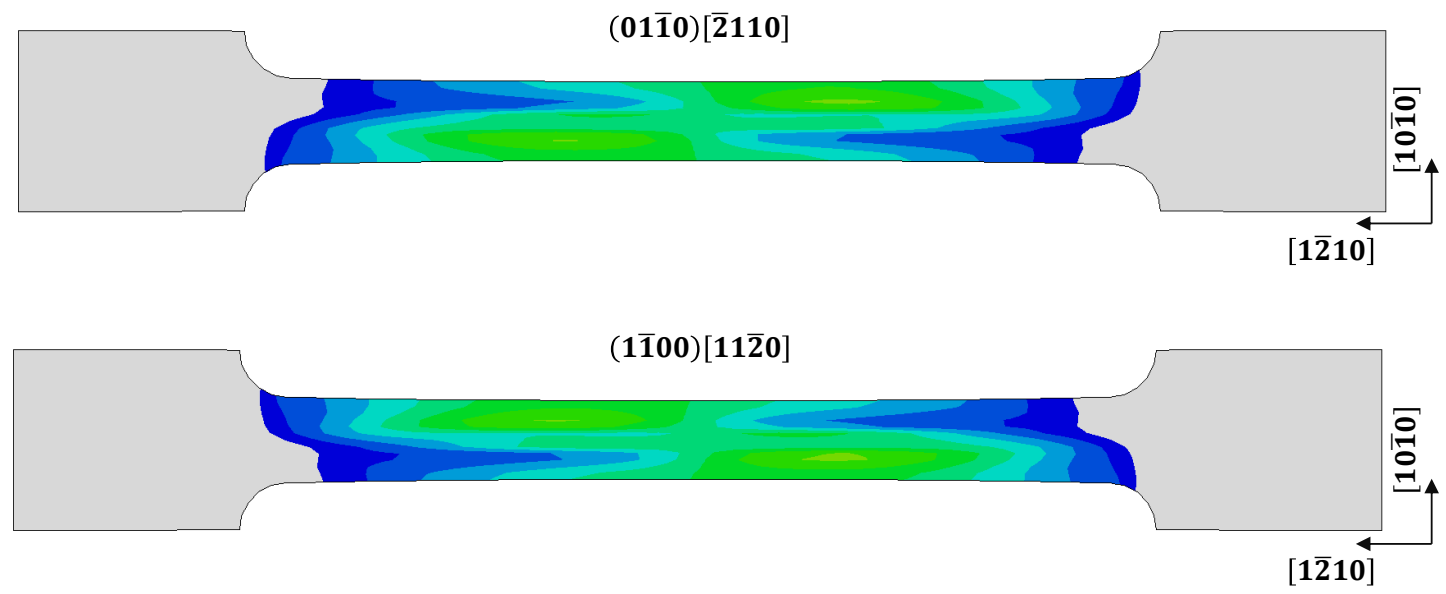

(a) Smooth

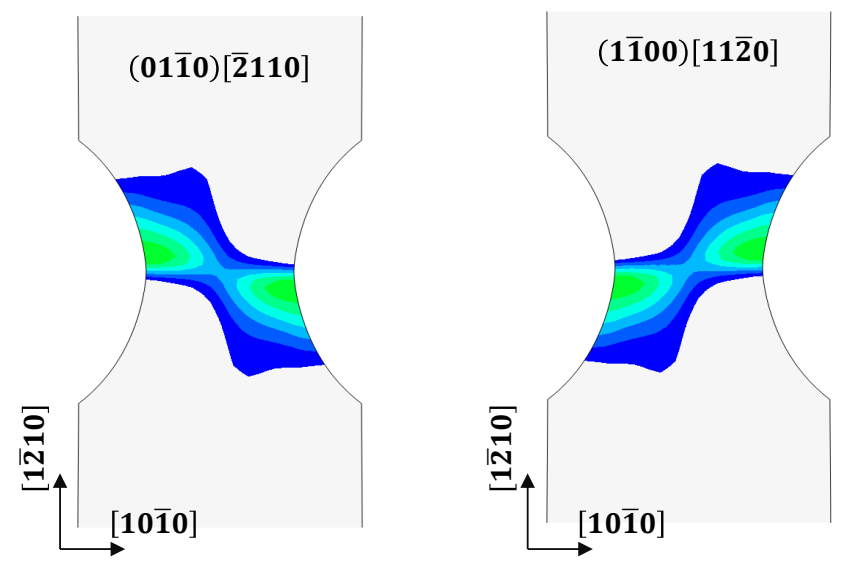

(b) RN10

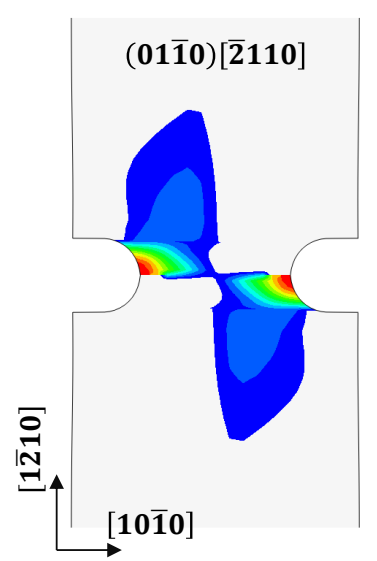

(c) $\mathrm{RN} 2$

Figure 11 Prismatic $\langle a\rangle$ slip in the (a) Smooth, (b) RN10 and (c) RN2 specimens of pure Mg single crystal. 


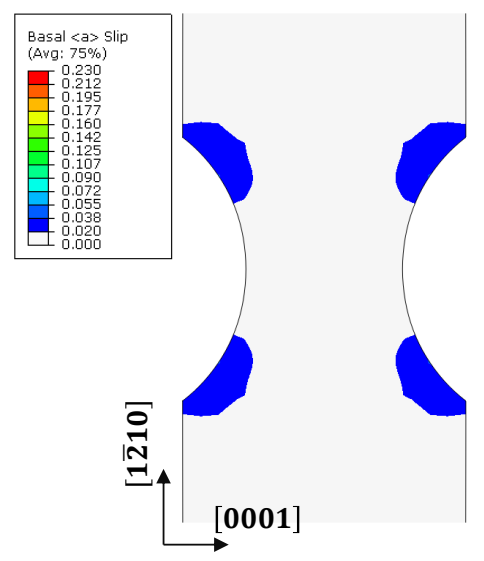

(a) RN10

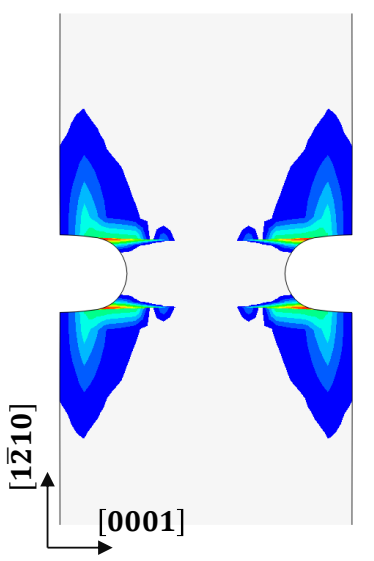

(b) RN2

Figure 12 Basal slip in the (a) RN10 and (b) RN2 specimens of pure Mg single crystal. Basal Slip is inactive in the smooth specimen since the Schmid factor is zero. 

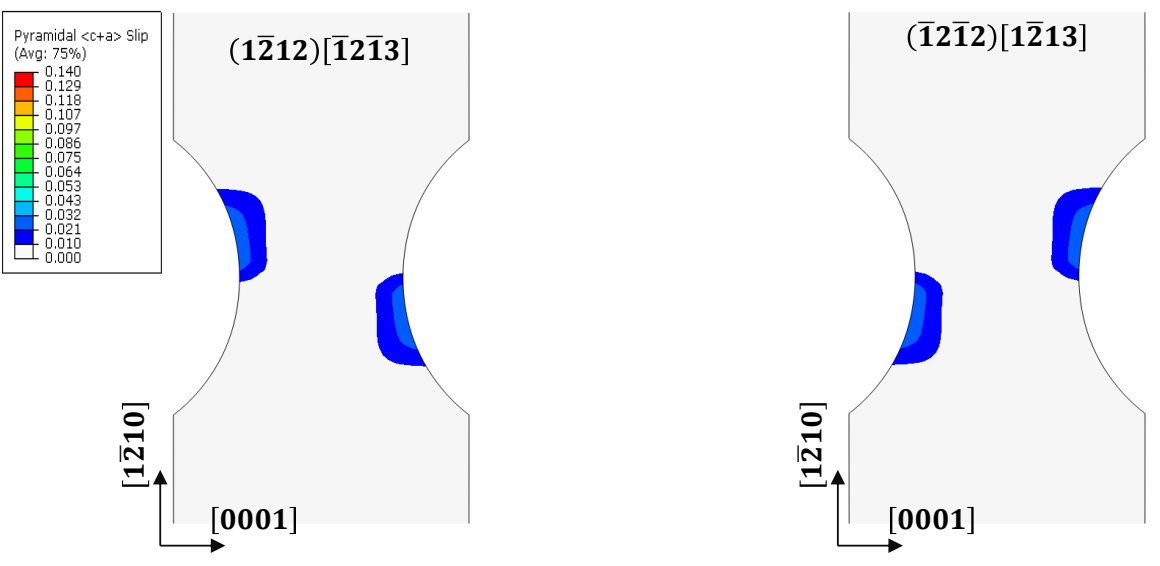

(a) RN10
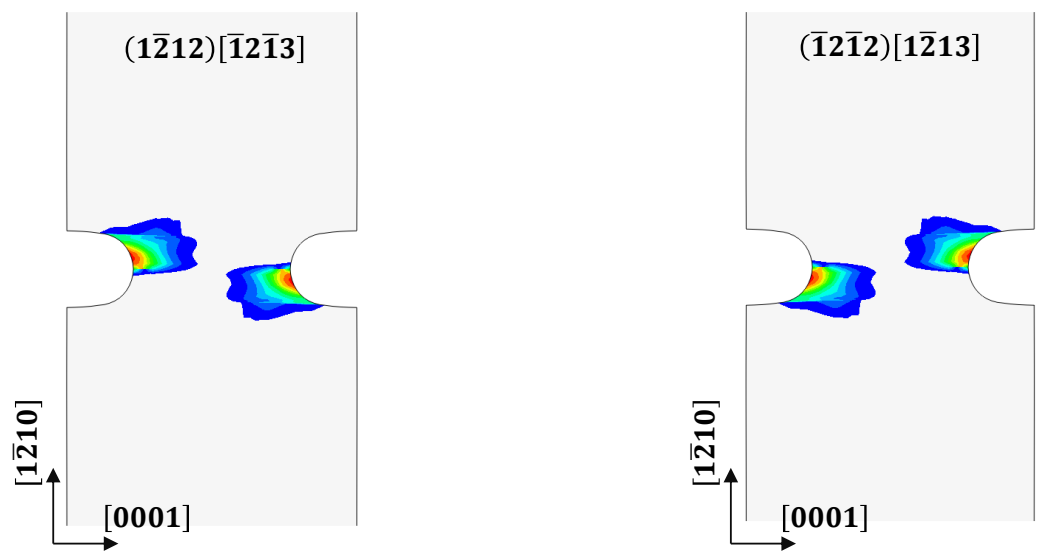

(b) RN2

Figure 13 Pyramidal $\langle c+a\rangle$ slip in the (a) RN10 and (b) RN2 specimens of pure Mg single crystal. Though the Schmid factor for pyramidal $\langle c+a\rangle$ slip in the smooth specimen is high, it is inactive due to latent hardening. 


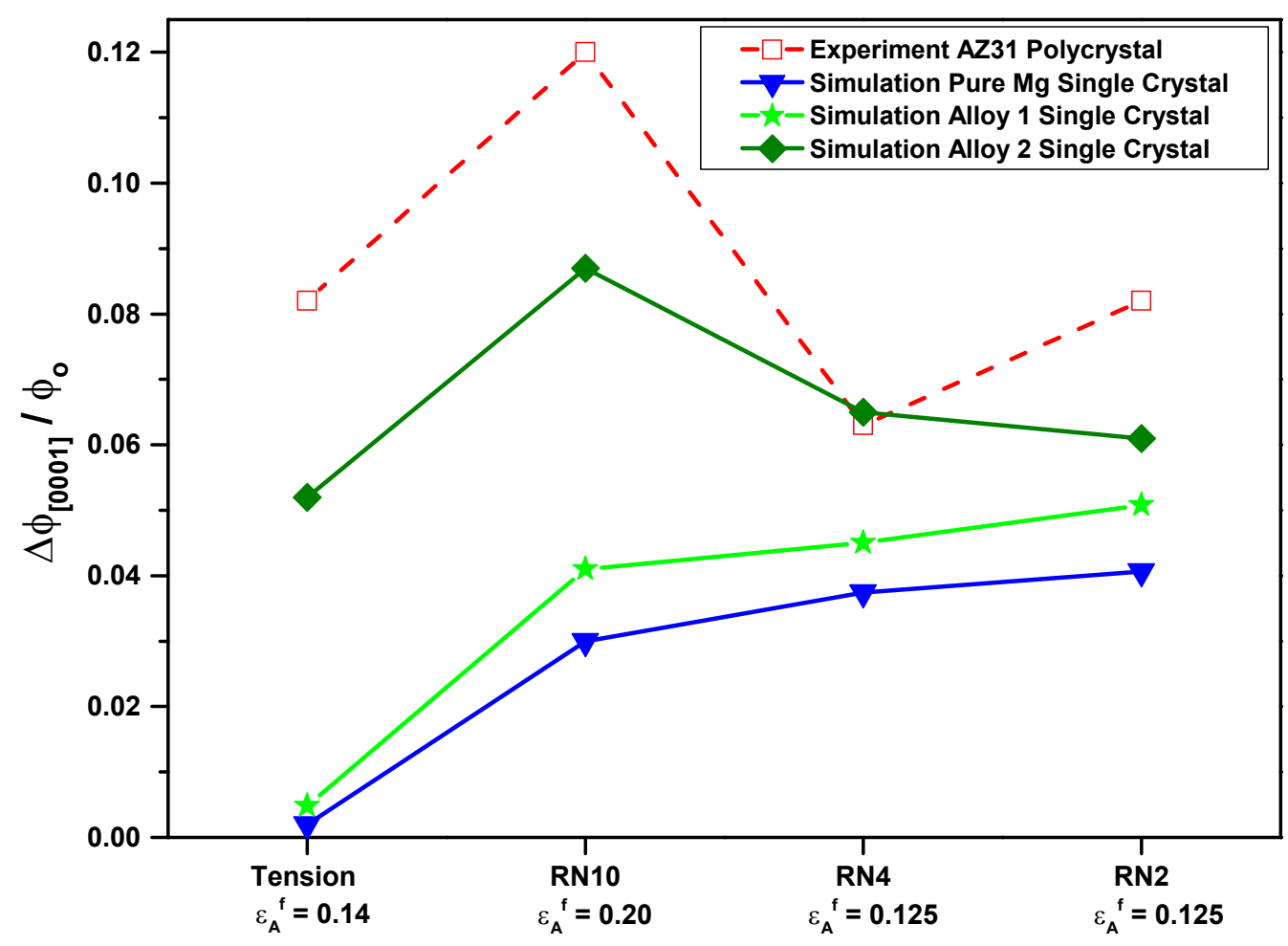

Figure 14 Comparison of diametric reduction at fracture initiation strain in the pure $\mathrm{Mg}$, alloy-1 and alloy-2 specimens with the experiments as function of triaxiality. 


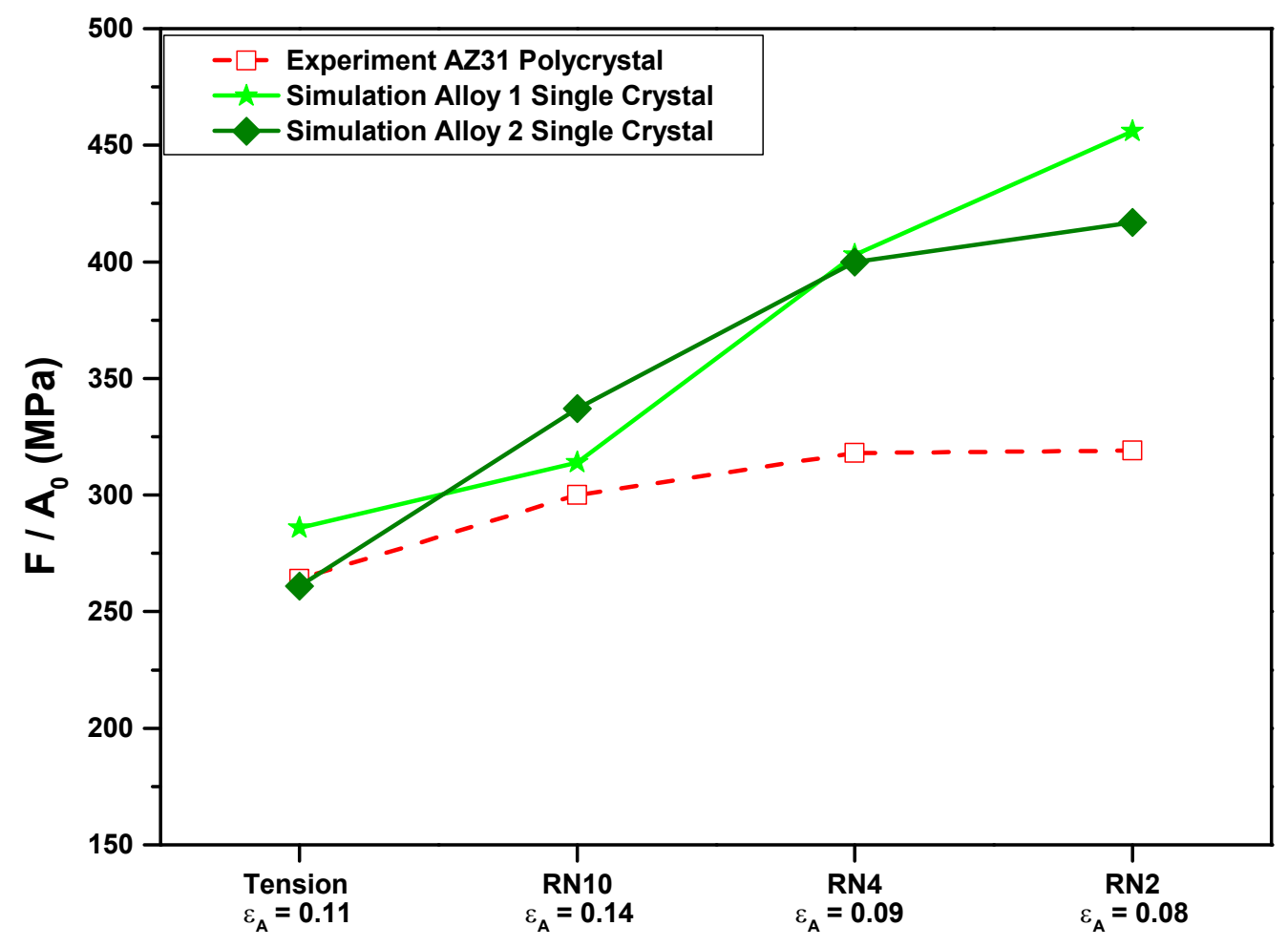

Figure 15 Comparison of load obtained from the simulations in the pure $\mathrm{Mg}$, alloy-1 and alloy-2 specimens with the limit load in the experiments as function of triaxiality. 


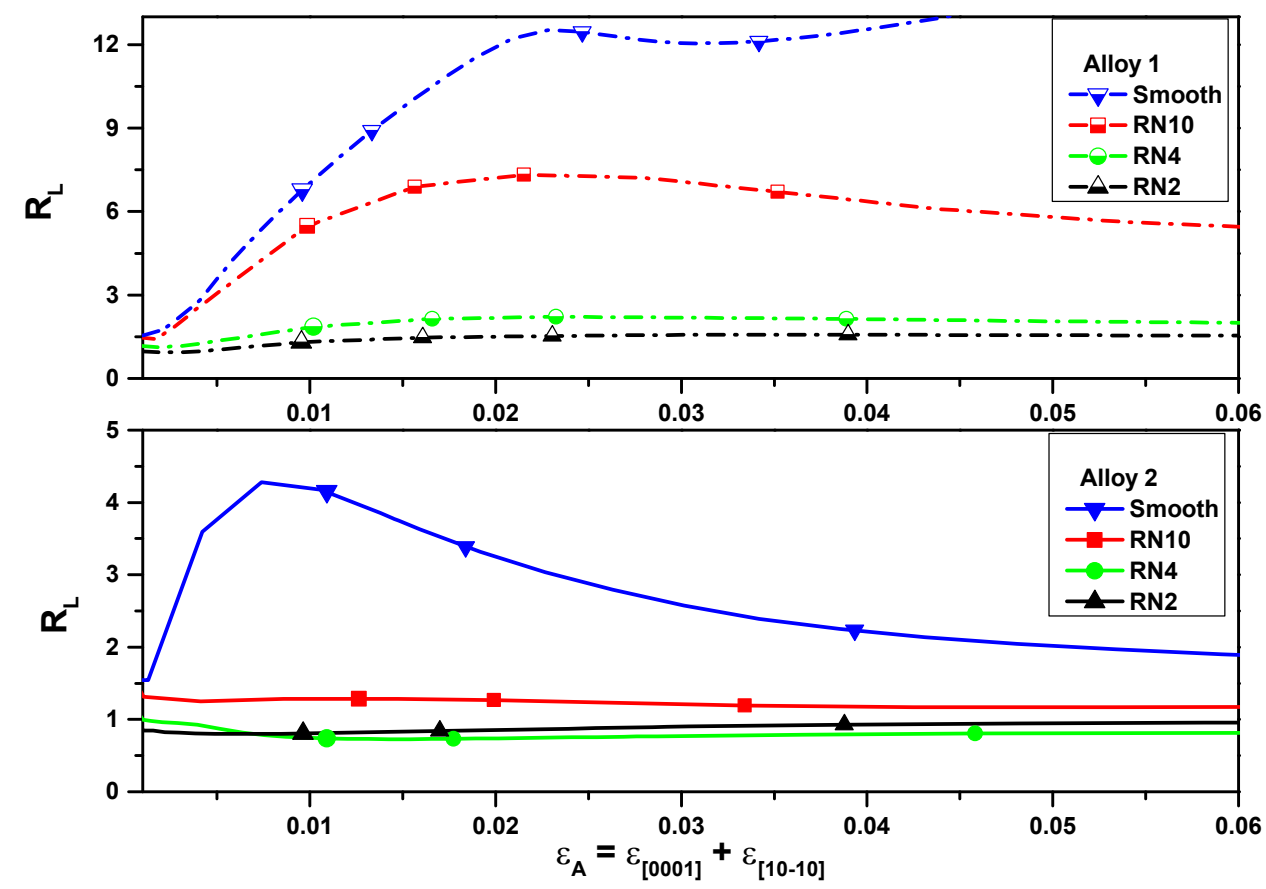

(a)

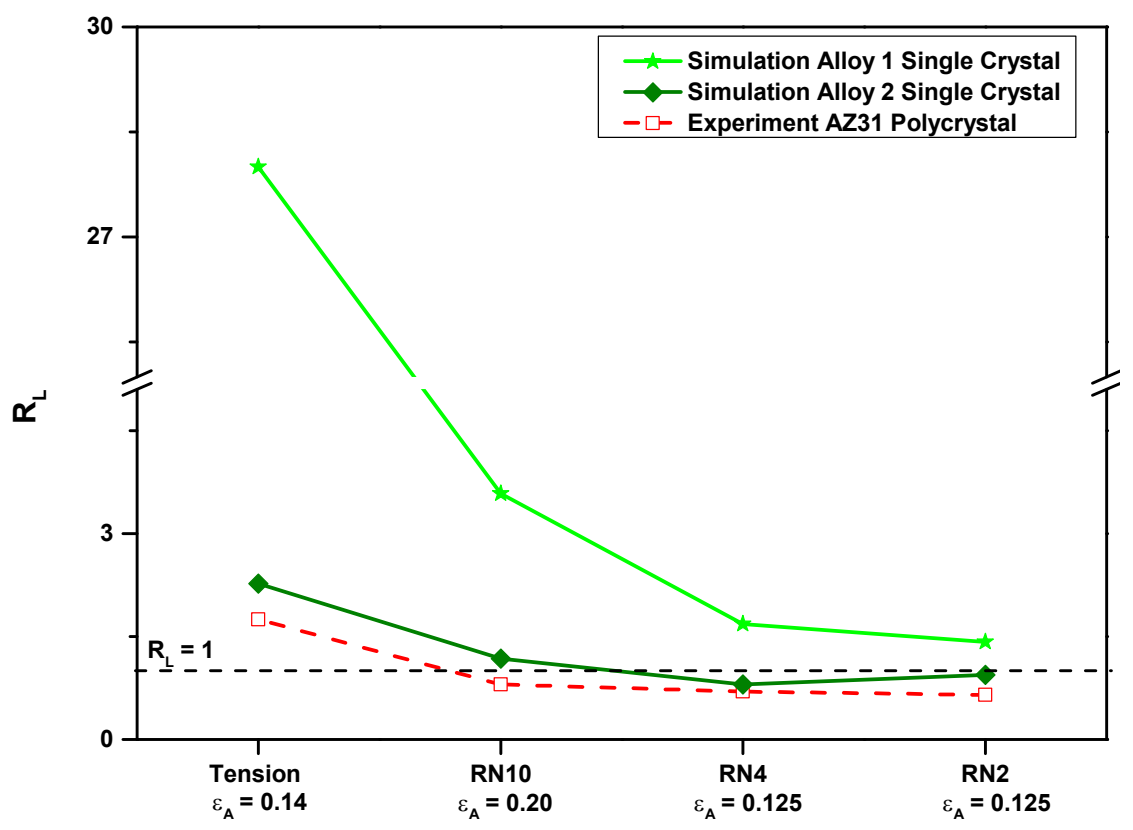

(b)

Figure 16 (a) Evolution of deformation anisotropy in the alloy-1 and alloy-2 specimens with overall areal strain, and (b) Comparison of $R_{\mathrm{L}}$ in the pure, alloy-1 and alloy-2 specimens with the experiments. The experimental $R_{L}$ values correspond to the fracture initiation strain and the same strain is used to extract

$R_{L}$ from the simulations. The dashed line in (b) represents $R_{L}$ for an isotropic material. 

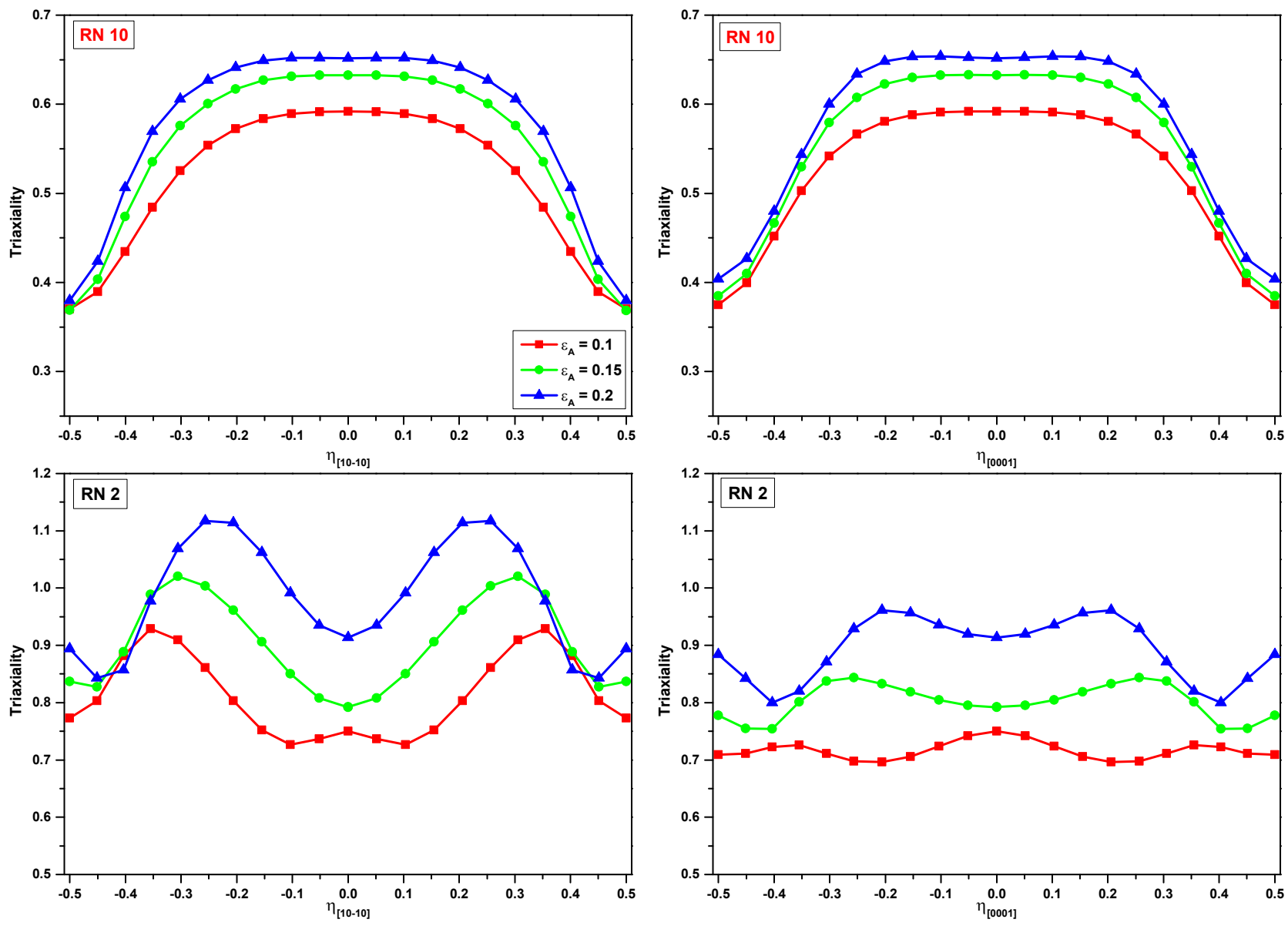

Figure 17 Triaxiality along [0001] and [1010] in the minimum section of alloy-2 single crystal 


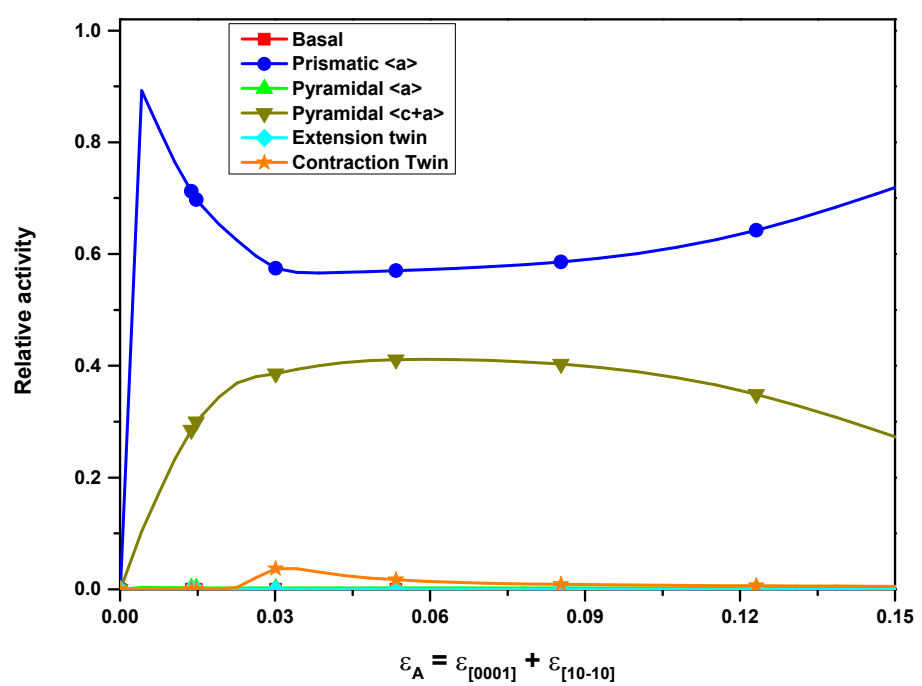

(a) Smooth

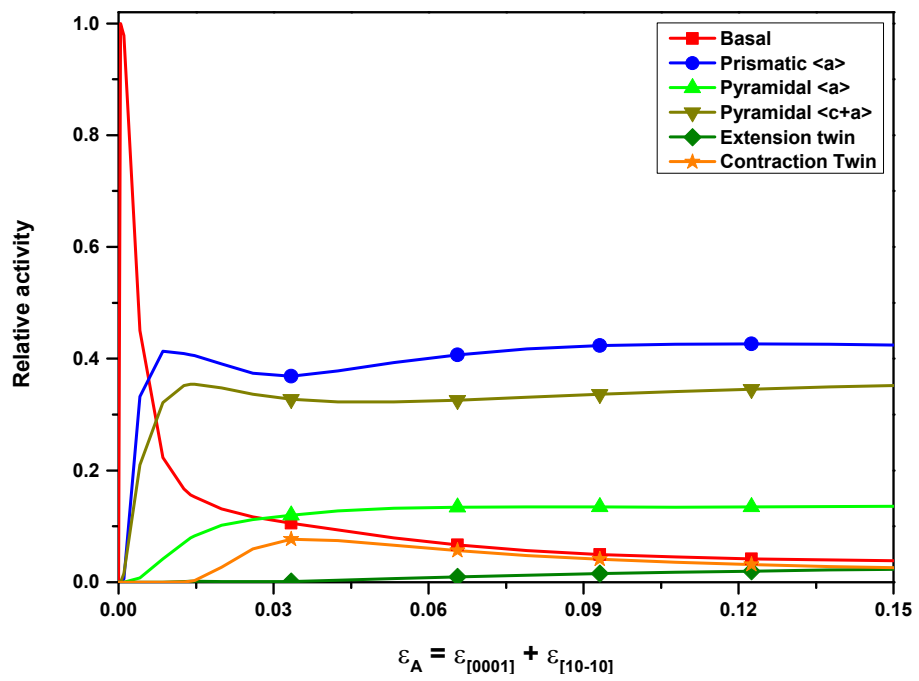

(b) RN10

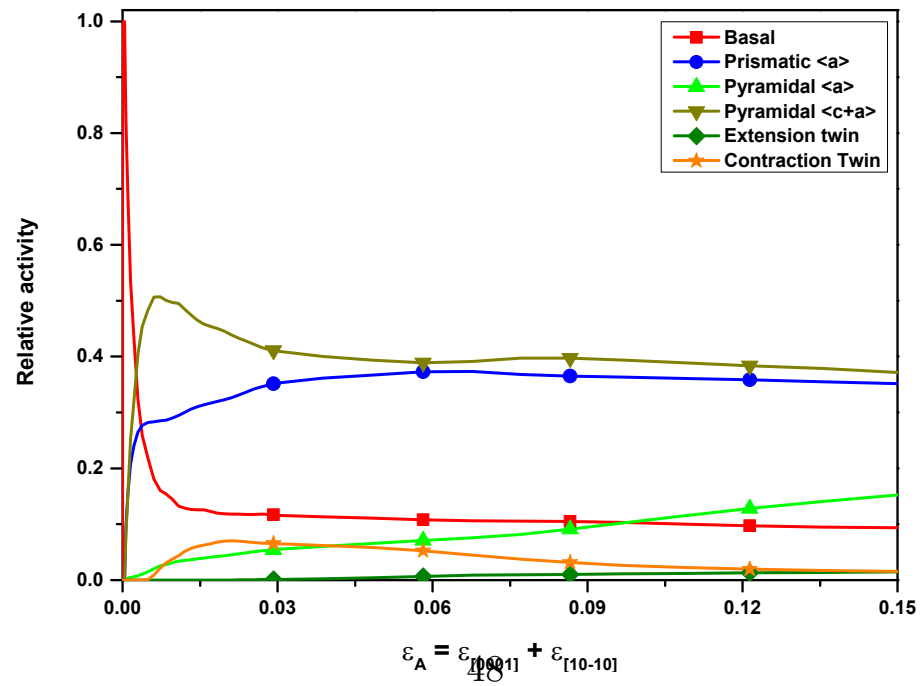

(c) RN2

Figure 18 Evolution of relative activity in (a) Smooth, (b) RN10 and (c) RN2 specimens of the alloy-2 

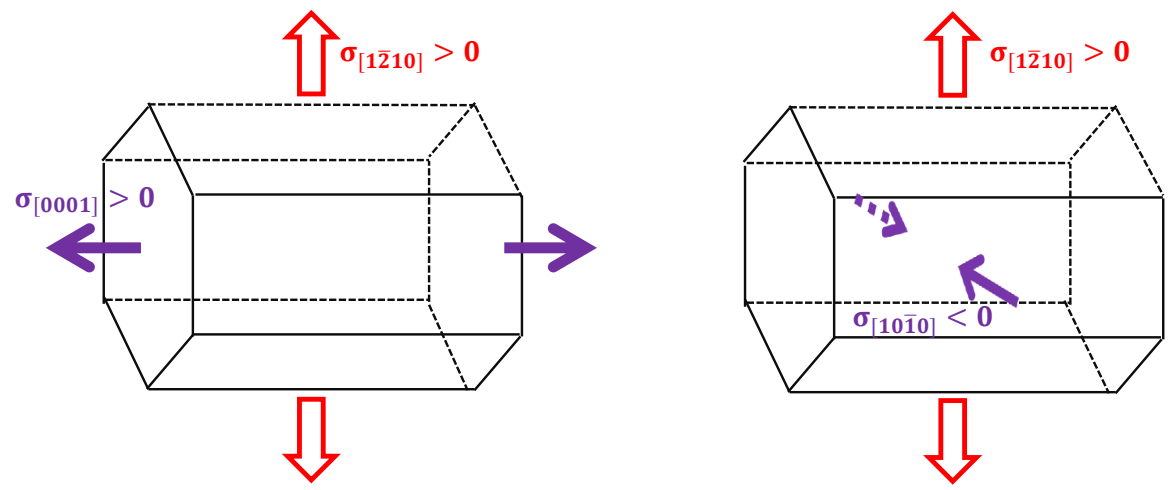

(a) Schematic of lateral normal stresses that aid extension twinning
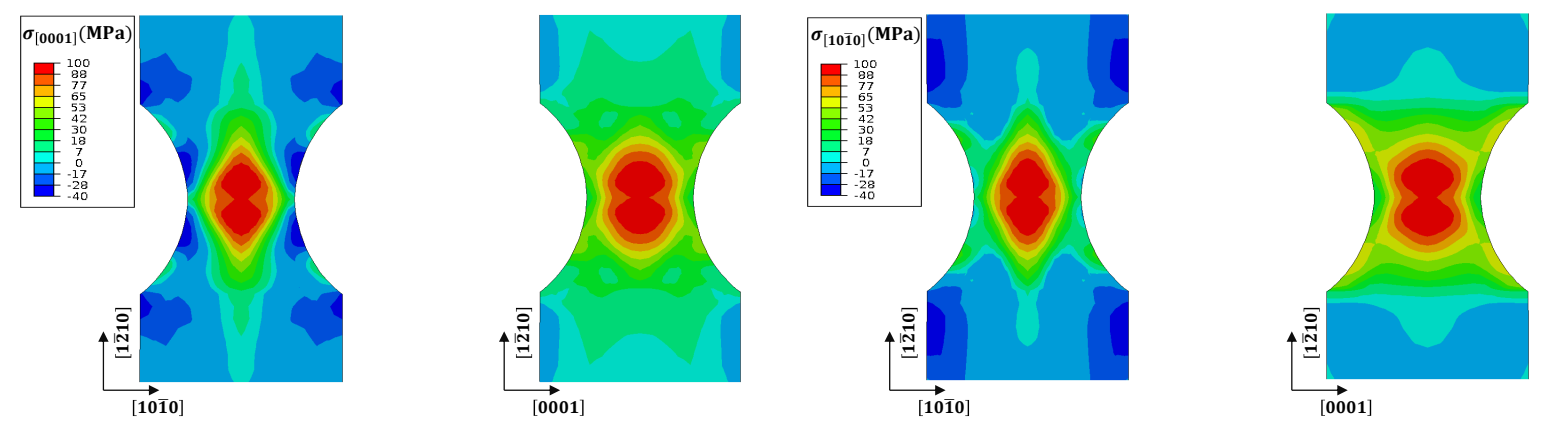

(b) $\sigma_{[0001]}$

(c) $\sigma_{[10 \overline{1} 0]}$
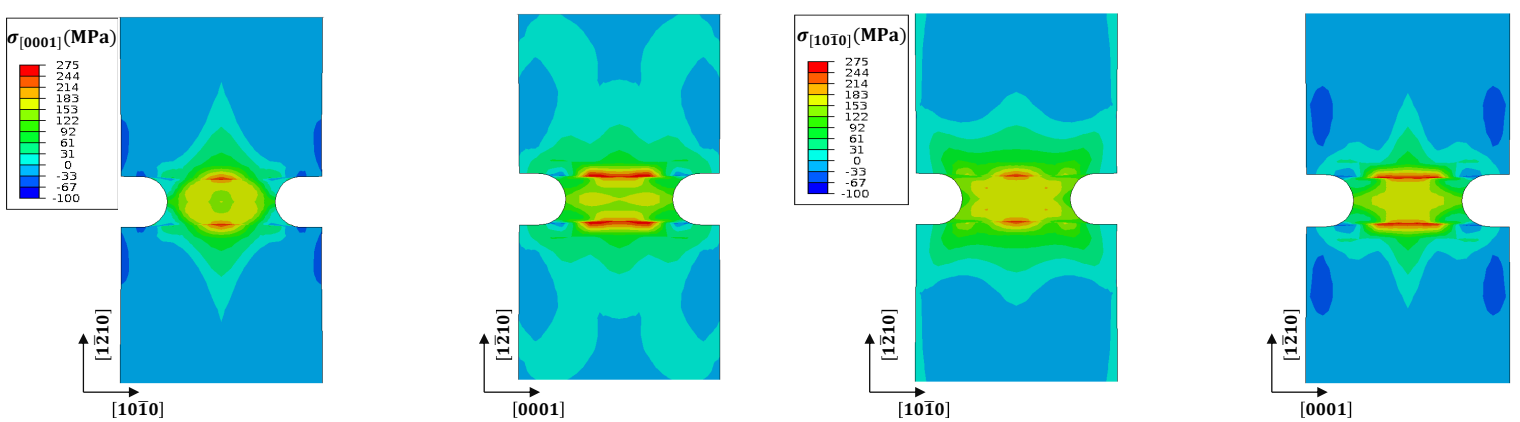

(d) $\sigma_{[0001]}$

(e) $\sigma_{[10 \overline{1} 0]}$

Figure 19 (a) Lateral normal stresses that promote extension twinning in the notch region. Panels (b-e) show the distribution of lateral stresses $\sigma_{[0001]}$ and $\sigma_{[10 \overline{1} 0]}$ in RN10 (b-c) and RN2 (d-e) specimens of alloy-2 single crystal at $\varepsilon_{\mathrm{A}}=0.1$ 

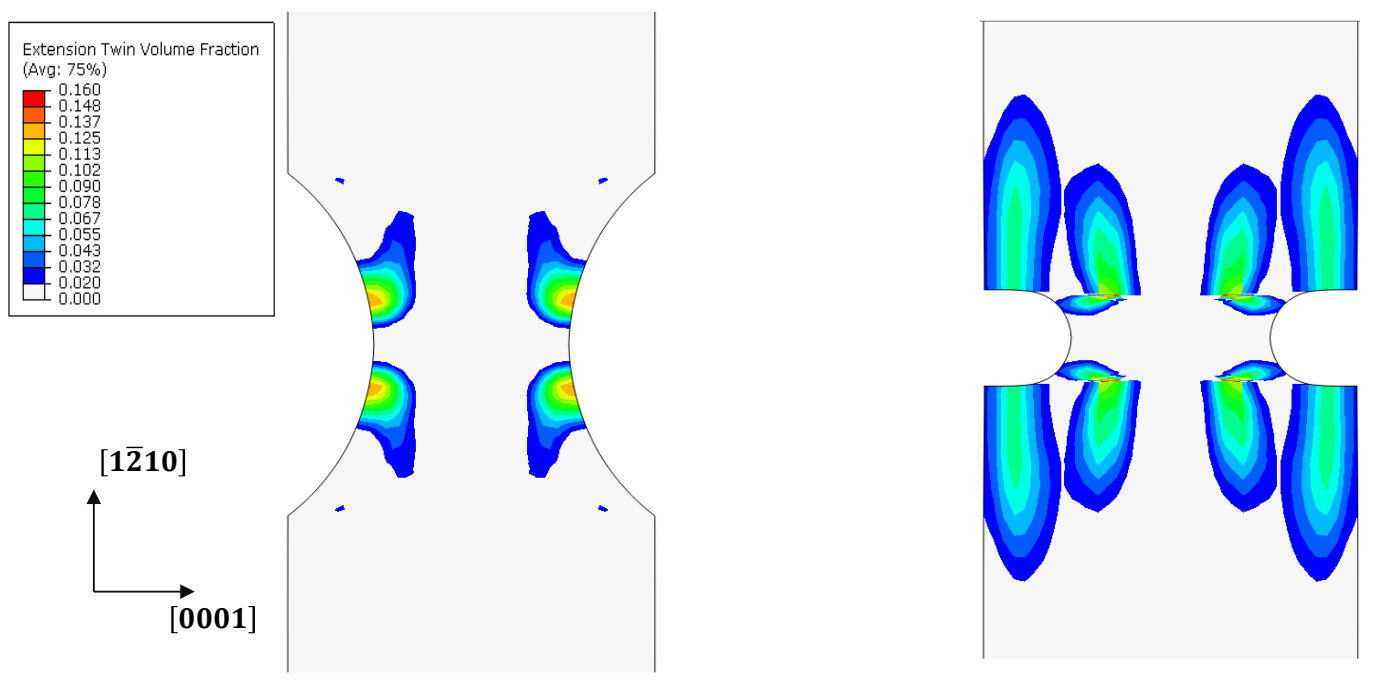

(a) RN10, $\varepsilon_{\mathrm{A}}=0.1$

(b) RN2, $\varepsilon_{\mathrm{A}}=0.1$

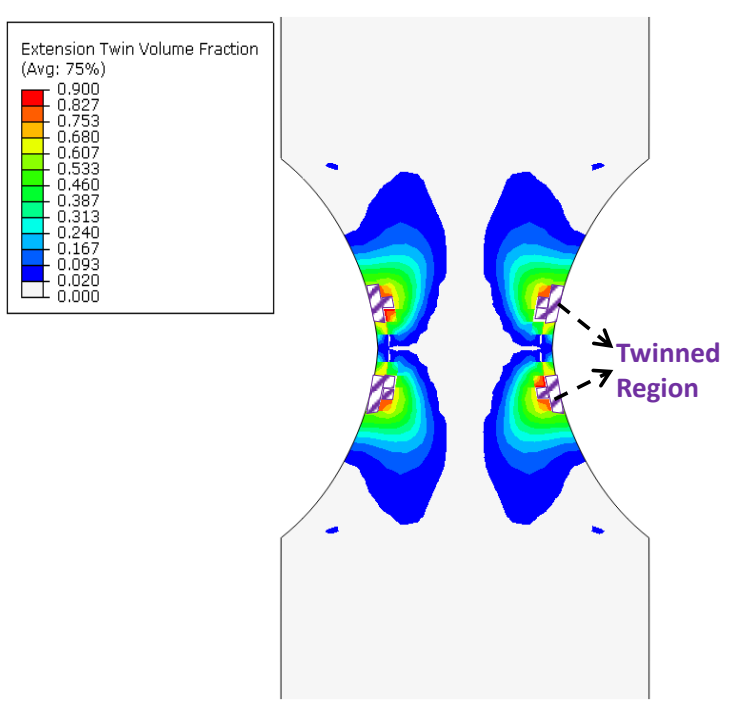

(c) $\mathrm{RN} 10, \varepsilon_{\mathrm{A}}=0.35$

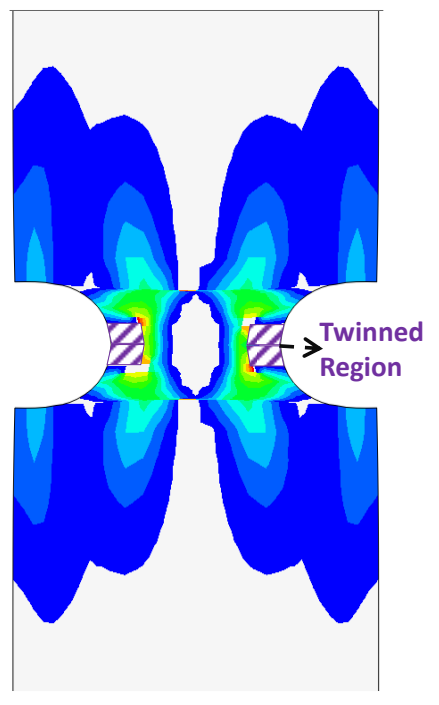

(d) $\mathrm{RN} 2, \varepsilon_{\mathrm{A}}=0.35$

Figure 20 Extension twin v.f. at $\varepsilon_{\mathbf{A}}=0.1$ in (a) RN10 (b) RN2, at $\varepsilon_{\mathbf{A}}=0.35$ in (c) RN10 (d) RN2 specimens of alloy-2 single crystal 


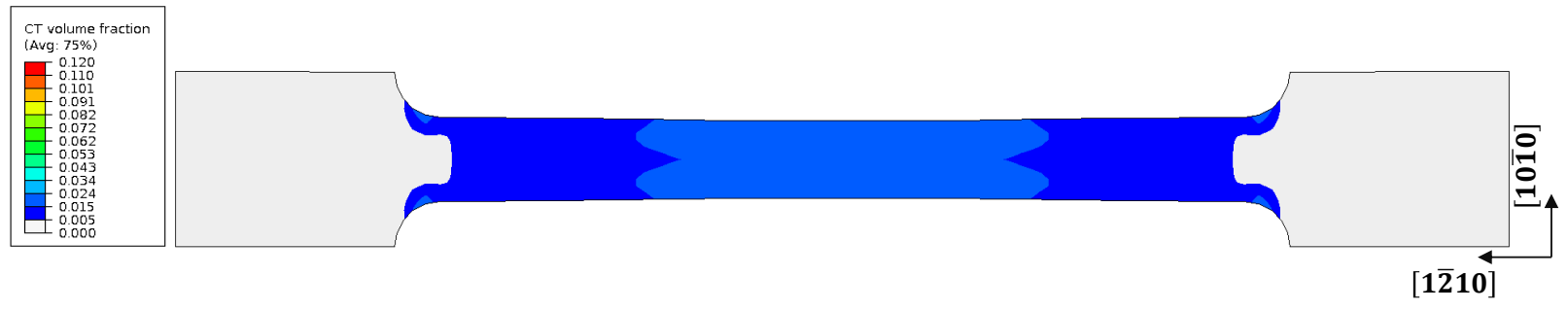

(a) Smooth

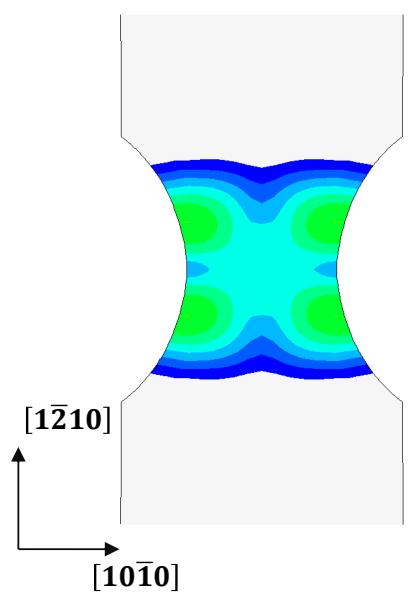

(b) RN10

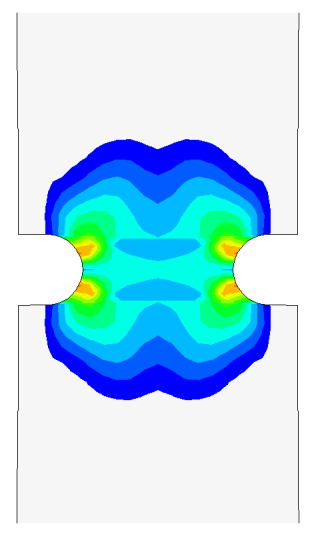

(c) RN2

Figure 21 Contraction twin v.f. in (a) Smooth, (b) RN10 (c) RN2 specimens of alloy-2 single crystal. $f^{\mathrm{CT}}<<f_{c}$ even at high values of strain. 


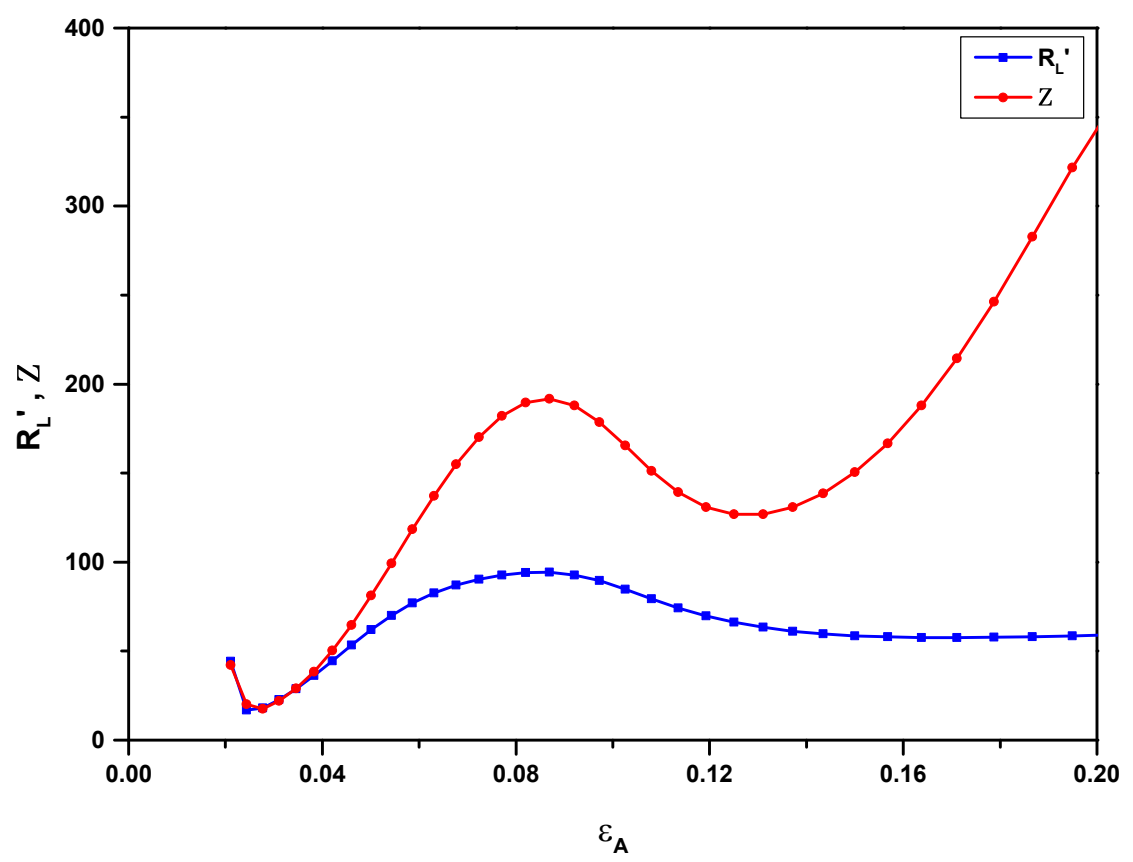

(a) Pure Mg

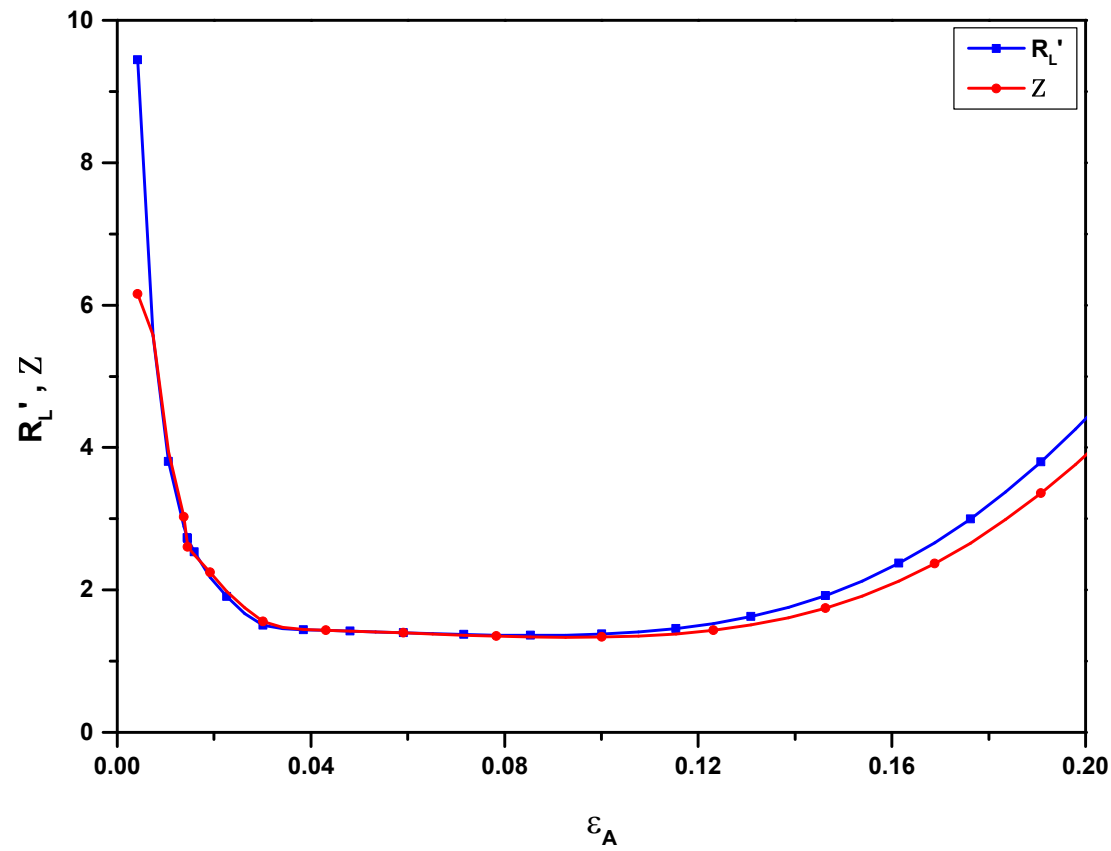

(b) Alloy-2

Figure 22 Comparison of $\mathrm{R}_{\mathrm{L}}{ }^{\prime}$ obtained from the simulations and $\mathcal{Z}$ calculated using the relative activity values. 